\title{
Static Aeroelastic and Longitudinal Trim Model of Flexible Wing Aircraft Using Finite-Element Vortex-Lattice Coupled Solution
}

\author{
Eric Ting* \\ Stinger Ghaffarian Technologies, Inc., Moffett Field, CA 94035 \\ Nhan Nguyen ${ }^{\dagger}$ \\ NASA Ames Research Center, Moffett Field, CA 94035 \\ Khanh Trinh ${ }^{\ddagger}$ \\ Stinger Ghaffarian Technologies, Inc., Moffett Field, CA 94035
}

\begin{abstract}
This paper presents a static aeroelastic model and longitudinal trim model for the analysis of a flexible wing transport aircraft. The static aeroelastic model is built using a structural model based on finite-element modeling and coupled to an aerodynamic model that uses vortex-lattice solution. An automatic geometry generation tool is used to close the loop between the structural and aerodynamic models. The aeroelastic model is extended for the development of a three degree-of-freedom longitudinal trim model for an aircraft with flexible wings. The resulting flexible aircraft longitudinal trim model is used to simultaneously compute the static aeroelastic shape for the aircraft model and the longitudinal state inputs to maintain an aircraft trim state. The framework is applied to an aircraft model based on the NASA Generic Transport Model (GTM) with wing structures allowed to flexibly deformed referred to as the Elastically Shaped Aircraft Concept (ESAC). The ESAC wing mass and stiffness properties are based on a baseline "stiff" values representative of current generation transport aircraft.
\end{abstract}

\section{Introduction}

In recent years, considerable attention within the aircraft design industry has been directed at incorporating lighter weight materials in the construction of aircraft structures. These efforts target reduction of aircraft weight, which translates into a lower lift requirement and in turn, reduces induced drag and thrust requirements. Usage of modern light-weight materials such as advanced composites has been adopted for use in the design of new aircraft. These materials are able to provide weight savings while maintaining the same load-carrying capacity as older material selections, also allowing the structural rigidity of the designs to be reduced. It becomes increasingly important for these modern designs to take into account the aeroelastic interactions between flight aerodynamics and the flexible aircraft structures within flight. Understanding and modeling these interactions can aid engineers in the analysis of design selections incorporating these lighter weight materials.

In 2010, a conceptual study titled "Elastically Shaped Future Air Vehicle Concept"1 was conducted to investigate the benefits of several advanced aircraft concepts over a conventional design. The study showed that there exists potential benefits in shaping wing surface aeroelastic deformation actively in flight with active control. The possibility of increasing aerodynamic efficiency by using active wing shaping for modern wing structures with reduced structural flexibility was realized. Development of modeling tools that can be used to investigate flexible wing aircraft and their aeroelastic behavior is emphasized in order to pursue these areas of research. These tools can also lead the way to flexible wing design optimization and the development of novel control surfaces to achieve active wing shaping, such as the Variable Camber Continuous Trailing Edge Flap system being investigated in a joint effort by NASA and Boeing. ${ }^{2,3}$

\footnotetext{
*Engineer, Intelligent Systems Division, eric.b.ting@ @asa.gov

${ }^{\dagger}$ Research Scientist, Intelligent Systems Division, nhan.t.nguyen@nasa.gov, AIAA Associate Fellow

‡Engineer, Intelligent Systems Division, khanh.v.trinh@ nasa.gov
} 
This paper outlines the development of a static aeroelasticity model and a three degree-of-freedom longitudinal trim model for an aircraft with flexible wing structures. The static aeroelasticity model utilizes a one-dimensional structural model of the the wing structure as a beam in coupled bending-torsion. ${ }^{4,5}$ The aeroelastic model also takes into account engine thrust forces and the effect of aero-propulsive-elasticity. ${ }^{5}$ Previous studies have utilized the Galerkin method ${ }^{4,6}$ to formulate a discretized weak-form solution to the structural equations. The similar numerical technique of finiteelement method (FEM) $)^{7-9}$ utilizing shape functions will be used in this study, as approached by previous work. ${ }^{5}$ An aeroelastic model is generated by coupling an aerodynamic model based off vortex-lattice data with the structural model through a geometry modeling tool that can create aeroelastically deformed aircraft models.

A three degree-of-freedom longitudinal trim model is developed as an extension of the static aeroelastic model. The aircraft's trim state is determined by balancing the longitudinal forces and moments on the flexible wing aircraft such that equilibrium is obtained. The trim model uses a standard Newton's method approach to solve the aircraft's equilibrium equations in the aircraft stability axes, while accounting for aeroelastic deflections. The modeling capability allows for the longitudinal states and the wing aeroelastic shape at trim to be determined. A comparison between a rigid wing aircraft and the effect of incorporating aeroelastic effects can be conducted.

The static aeroelasticity and the three degree-of-freedom longitudinal trim model are applied to various different aircraft models. The models are based on the NASA Generic Transport Model (GTM), which is a transport aircraft airframe similar in class to the Boeing 757. ${ }^{10}$ A simplified model based upon the planform and the characteristics of the jig-shape wing is developed, called the "Idealized Wing Alone" model. Validation of the developed static aeroelastic framework is conducted using the Idealized Wing Alone model and the results are compared against NASTRAN aeroelastic results provided by Boeing Research and Technology. ${ }^{2}$ A full aircraft model with fuselage, tails, and engines referred to as the Elastically Shaped Aircraft Concept (ESAC) is utilized. The ESAC model can utilize a rigid wing model or wing model where aeroelastic deformation is considered. For the flexible wing model, the ESAC model utilizes mass and stiffness values representative of the GTM wing. The static aeroelastic framework is applied to the ESAC, and the resulting model is extended to develop the trim solution capable of handling wing aeroelasticity.

\section{Aircraft Models}

\section{A. Elastically Shaped Aircraft Concept}

The Elastically Shaped Aircraft Concept (ESAC) is a model of a complete, typical, transport aircraft configuration. It is developed based upon the NASA Generic Transport Model (GTM) ${ }^{10}$ which represents a notional single-aisle, mid-size, 200-passenger aircraft. The GTM is a research platform that includes a wind tunnel model and a remotely piloted vehicle. An extensive wind tunnel aerodynamic database ${ }^{10}$ also exists for the GTM configuration lending itself for understanding and validation of results. Figure 1 is an illustration of the GTM planform. The benchmark configuration represents one of the most common types of transport aircraft in the commercial aviation sector that provides short-to-medium range, 3000 nautical mile, passenger carrying capacities.

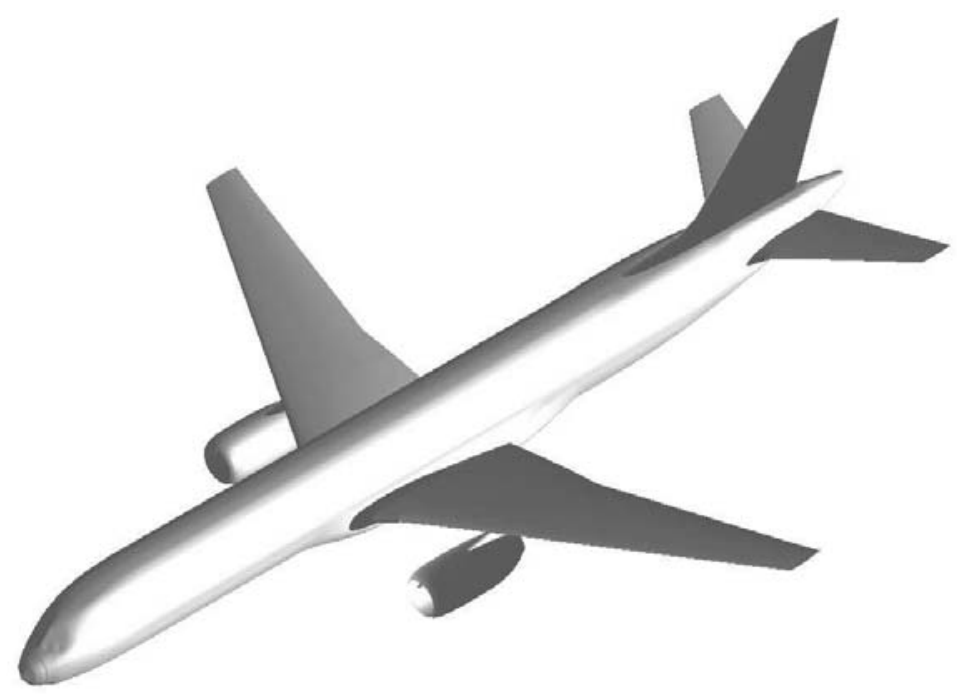

Figure 1. Benchmark GTM Planform 
The geometry of the ESAC is obtained by scaling up the geometry of GTM wind tunnel model by a scale of 200:11. In the aeroelastic model, the jig-shape wings of the ESAC can be analyzed using a rigid model or be allowed to freely deform based on reference wing stiffness values. The equivalent beam model for the wing and the aircraft mass and baseline stiffness values are built using a component-based approach. The aircraft is divided into the following components: fuselage, wings, horizontal tails, vertical tail, engines, operational empty weight (or OEW equipment), and typical load including passengers, cargo, and fuel. The fuselage, wings, horizontal tails, and vertical tail are modeled as shell structures with constant wall thicknesses. ${ }^{11}$

\section{B. Idealized Wing Alone Planform Model}

Development of the Idealized Wing Alone model is conducted using the undeformed jig-shape wing of the ESAC. Using the geometric wing pre-twist, measurements of mean camber line, and planform shape, the removal of the fuselage, tails, engines, and pylons allows for the construction of the wing alone model. This wing alone model is idealized as a surface with no thickness, and the wing root is extended from the planform of the original wing to the aircraft centerline. The idealized surface can then be twisted based on the jig-shape geometry wing pre-twist, or shaped based on camber line measurements. The resulting Idealized Wing Alone model represents only a wing surface, and the jig-shape's inherent twist and camber can be individually activated on the model for incremental analysis of their effect on static aeroelasticity.

Figure 2 is a depiction of the Idealized Wing Alone model within the vortex-lattice modeling program Vorview.

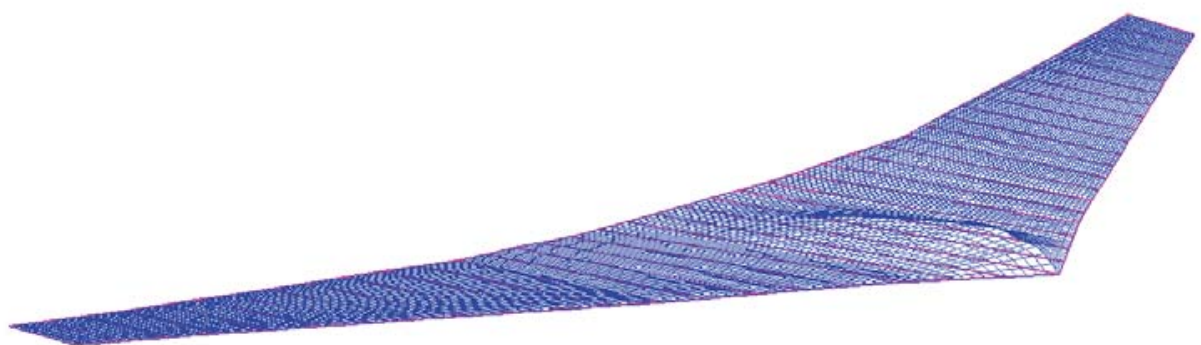

Figure 2. Idealized Wing Along Planform In Vorview

\section{Wing Structural Modeling}

A structural model of the wing using beam theory is developed and is later incorporated into a fully coupled structural-aerodynamic aeroelasticity model. The model is similar to those developed previously in other studies. ${ }^{4,5}$

\section{A. Reference Frames}

Figure 3 illustrates three orthogonal views for a typical transport aircraft and several associated reference frames. These reference frames are useful in developing the structural models of the lifting surfaces of an aircraft, although the coordinate frames associated with the aircraft wings are primarily used in this analysis. The aircraft body-fixed reference frame $B$ is defined by the unit vectors $\boldsymbol{b}_{1}, \boldsymbol{b}_{2}$, and $\boldsymbol{b}_{3}$, which are aligned with the aircraft roll, pitch, and yaw axes, respectively. 


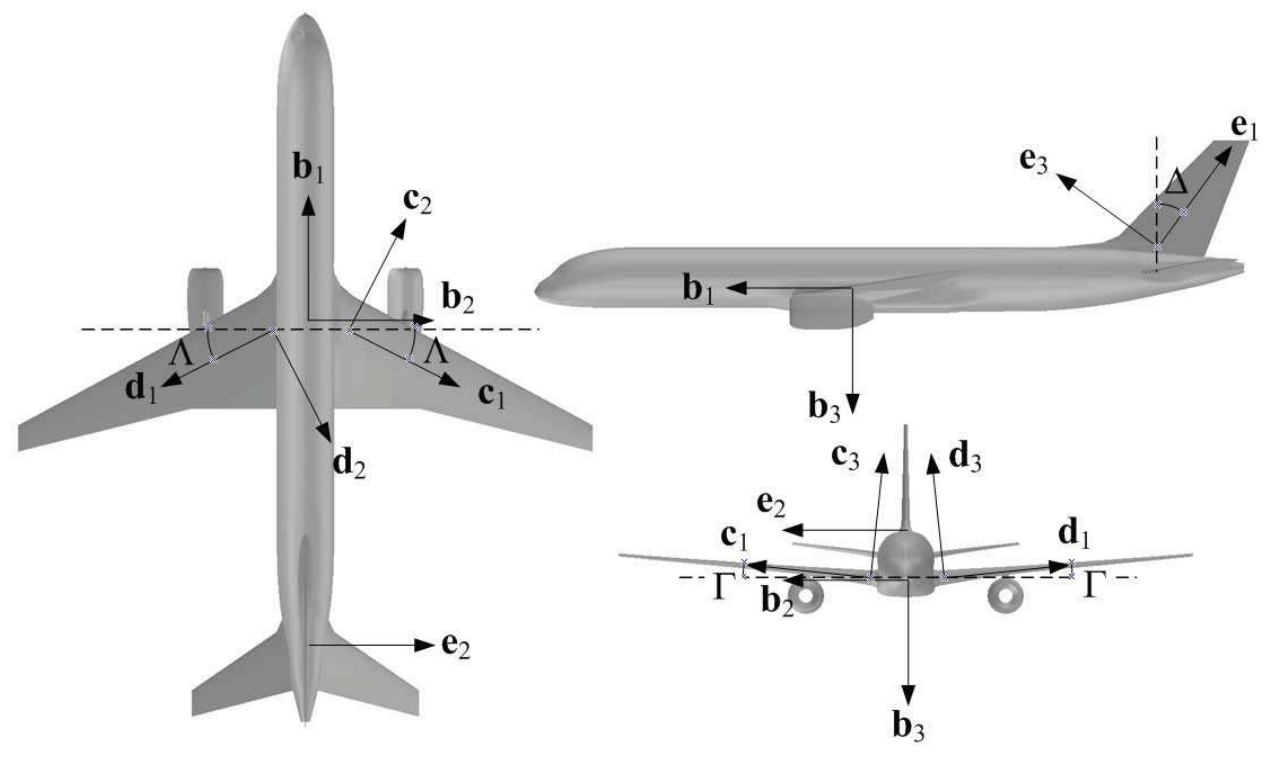

Figure 3. Aircraft Reference Frames

The reference frame $C$ is aligned with the right wing's elastic axis and is defined by the unit vectors $\boldsymbol{c}_{1}, \boldsymbol{c}_{2}$, and $c_{3}$. Let $\Lambda$ be the sweep of the wing's elastic axis. The $B$ frame can be related to $C$ through three successive rotations: 1) the first rotation about $\boldsymbol{b}_{3}$ by an angle of $\frac{\pi}{2}+\Lambda$ to generate an intermediate reference frame $B^{\prime}$ defined by the unit vectors $\boldsymbol{b}_{1}^{\prime}, \boldsymbol{b}_{2}^{\prime}$, and $\boldsymbol{b}_{3}^{\prime}$ (not shown), 2) the second rotation about $\boldsymbol{b}_{2}^{\prime}$ by the dihedral angle $\Gamma$ of the elastic axis that results in the intermediate reference frame $C^{\prime}$ defined by the unit vectors $\boldsymbol{c}_{1}^{\prime}, \boldsymbol{c}_{2}^{\prime}$, and $\boldsymbol{c}_{3}^{\prime}$ (not shown), and 3 ) the third rotation about $c_{1}^{\prime}$ by an angle of $\pi$ to result in the reference frame $C$. The transformation can be represented by a series of coordinate rotations as

$$
\begin{aligned}
{\left[\begin{array}{l}
\boldsymbol{b}_{1} \\
\boldsymbol{b}_{2} \\
\boldsymbol{b}_{3}
\end{array}\right]=} & {\left[\begin{array}{ccc}
-\sin \Lambda & -\cos \Lambda & 0 \\
\cos \Lambda & -\sin \Lambda & 0 \\
0 & 0 & 1
\end{array}\right]\left[\begin{array}{ccc}
\cos \Gamma & 0 & \sin \Gamma \\
0 & 1 & 0 \\
-\sin \Gamma & 0 & \cos \Gamma
\end{array}\right]\left[\begin{array}{ccc}
1 & 0 & 0 \\
0 & -1 & 0 \\
0 & 0 & -1
\end{array}\right]\left[\begin{array}{l}
\boldsymbol{c}_{1} \\
\boldsymbol{c}_{2} \\
\boldsymbol{c}_{3}
\end{array}\right] } \\
= & {\left[\begin{array}{ccc}
-\sin \Lambda \cos \Gamma & \cos \Lambda & \sin \Lambda \sin \Gamma \\
\cos \Lambda \cos \Gamma & \sin \Lambda & -\cos \Lambda \sin \Gamma \\
-\sin \Gamma & 0 & -\cos \Gamma
\end{array}\right]\left[\begin{array}{l}
\boldsymbol{c}_{1} \\
\boldsymbol{c}_{2} \\
\boldsymbol{c}_{3}
\end{array}\right] }
\end{aligned}
$$

The analysis can be repeated for the left wing. The reference frame $D$ is aligned with the left wing's elastic axis and is defined by the unit vectors $\boldsymbol{d}_{1}, \boldsymbol{d}_{2}$, and $\boldsymbol{d}_{3}$. The $B$ frame can be related to $D$ through three successive rotations: 1) the first rotation about $-\boldsymbol{b}_{3}$ by an angle of $\frac{\pi}{2}+\Lambda$ to generate an intermediate reference frame $B^{\prime \prime}$ defined by the unit vectors $\boldsymbol{b}_{1}^{\prime \prime}, \boldsymbol{b}_{2}^{\prime \prime}$, and $\boldsymbol{b}_{3}^{\prime \prime}$ (not shown), 2) the second rotation about $\boldsymbol{b}_{2}^{\prime \prime}$ by the dihedral angle $\Gamma$ of the elastic axis that results in the intermediate reference frame $D^{\prime}$ defined by the unit vectors $\boldsymbol{d}_{1}^{\prime}, \boldsymbol{d}_{2}^{\prime}$, and $\boldsymbol{d}_{3}^{\prime}$ (not shown), and 3) the third rotation about $\boldsymbol{d}_{1}^{\prime}$ by an angle of $\pi$ to result in the reference frame $D$. The relationship can be expressed as

$$
\begin{aligned}
{\left[\begin{array}{l}
\boldsymbol{b}_{1} \\
\boldsymbol{b}_{2} \\
\boldsymbol{b}_{3}
\end{array}\right]=} & {\left[\begin{array}{ccc}
-\sin \Lambda & \cos \Lambda & 0 \\
-\cos \Lambda & -\sin \Lambda & 0 \\
0 & 0 & 1
\end{array}\right]\left[\begin{array}{ccc}
\cos \Gamma & 0 & \sin \Gamma \\
0 & 1 & 0 \\
-\sin \Gamma & 0 & \cos \Gamma
\end{array}\right]\left[\begin{array}{ccc}
1 & 0 & 0 \\
0 & -1 & 0 \\
0 & 0 & -1
\end{array}\right]\left[\begin{array}{l}
\boldsymbol{d}_{1} \\
\boldsymbol{d}_{2} \\
\boldsymbol{d}_{3}
\end{array}\right] } \\
= & {\left[\begin{array}{ccc}
-\sin \Lambda \cos \Gamma & -\cos \Lambda & \sin \Lambda \sin \Gamma \\
-\cos \Lambda \cos \Gamma & \sin \Lambda & \cos \Lambda \sin \Gamma \\
-\sin \Gamma & 0 & -\cos \Gamma
\end{array}\right]\left[\begin{array}{l}
\boldsymbol{d}_{1} \\
\boldsymbol{d}_{2} \\
\boldsymbol{d}_{3}
\end{array}\right] }
\end{aligned}
$$

\section{B. Elastic Axis}

An analysis of the combined motion of the left wing is conducted in the present section, and the motion of the right wing is considered to be equivalent for symmetric flight. This analysis is equivalent to that in a previous study ${ }^{5}$ and is 
included for completeness.

Let $x$ represent the coordinate along the elastic axis of a wing running from root to tip. The wing pre-twist angle $\gamma(x)$ thus represents the incidence of the airfoil section at the corresponding elastic axis coordinate. A typical wing pretwist varies from nose-up at the wing root to nose-down at the wing tip and is commonly referred to as a "wash-out" twist distribution.

The internal structure of a wing is typically composed of a complex arrangement of load carrying spars and wing boxes that carry the stresses and strains introduced by aerodynamic forces and aeroelastic deflections. For this analysis, an equivalent beam approach is used which models the wing's elastic behavior using equivalent stiffness properties. It is a common approach in analyzing aeroelastic deflections ${ }^{7}$ and can be used to analyze high aspect ratio wings with good accuracy. The effect of wing curvature is ignored and straight beam theory is used to model the wing deflection. The axial or extensional deflection of a wing is also generally very small and is neglected.

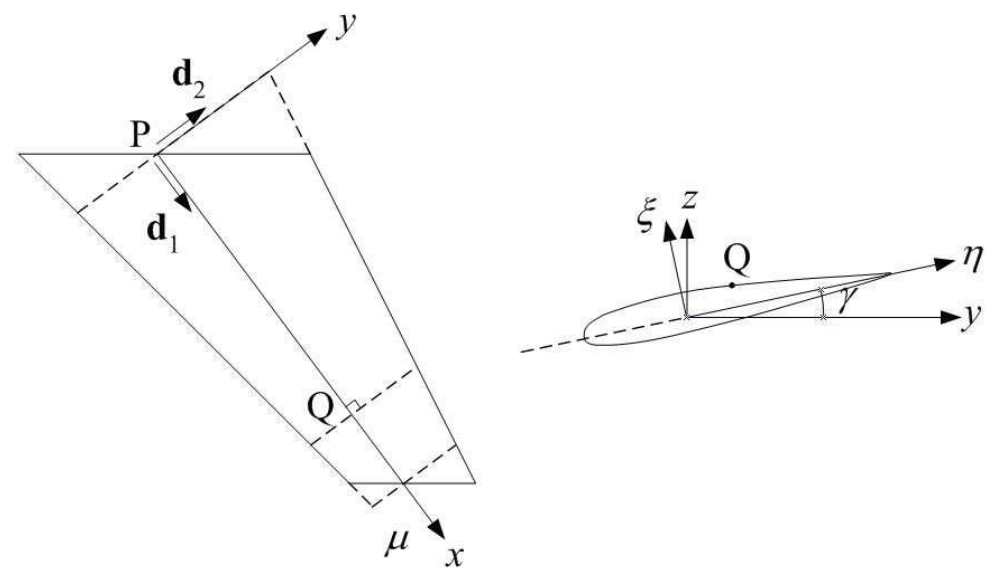

Figure 4. Left Wing Reference Frame

Consider an airfoil section on the left wing as shown in Fig. 4 undergoing bending and torsional deflections. Let $(x, y, z)$ be the coordinates of point $\mathrm{Q}$ on the wing airfoil section. The undeformed local airfoil coordinates of point $\mathrm{Q}$ are

$$
\left[\begin{array}{l}
y \\
z
\end{array}\right]=\left[\begin{array}{cc}
\cos \gamma & -\sin \gamma \\
\sin \gamma & \cos \gamma
\end{array}\right]\left[\begin{array}{l}
\eta \\
\xi
\end{array}\right]
$$

where $\eta$ and $\xi$ are the local airfoil coordinates, and $\gamma$ is the wing section pre-twist angle, positive nose-down. ${ }^{12}$ The wing pre-twist is defined with respect to the elastic axis of the wing.

Differentiating with respect to $x$ gives

$$
\left[\begin{array}{l}
y_{x} \\
z_{x}
\end{array}\right]=\gamma^{\prime}\left[\begin{array}{cc}
-\sin \gamma & -\cos \gamma \\
\cos \gamma & -\sin \gamma
\end{array}\right]\left[\begin{array}{l}
\eta \\
\xi
\end{array}\right]=\left[\begin{array}{c}
-z \gamma^{\prime} \\
y \gamma^{\prime}
\end{array}\right]
$$

Let $\Theta$ be a torsional twist angle about the x-axis, positive nose-down, and let $W$ and $V$ be flapwise and chordwise bending deflections of point $\mathrm{Q}$, respectively. Then, the rotation angle due to the elastic deformation can be expressed as

$$
\boldsymbol{\phi}(x, t)=\Theta \boldsymbol{d}_{1}-W_{x} \boldsymbol{d}_{2}+V_{x} \boldsymbol{d}_{3}
$$

where the subscript $x$ denotes the partial derivatives of $\Theta, W$, and $V$ with respect to $x$.

Let $\left(x_{1}, y_{1}, z_{1}\right)$ be the coordinates of point $\mathrm{Q}$ on the airfoil in the reference frame $D$ with aeroelastic deformation. The coordinates $\left(x_{1}, y_{1}, z_{1}\right)$ are computed using the small angle approximation as

$$
\left[\begin{array}{c}
x_{1}(x, t) \\
y_{1}(x, t) \\
z_{1}(x, t)
\end{array}\right]=\left[\begin{array}{c}
x \\
y+V \\
z+w
\end{array}\right]+\left[\begin{array}{c}
\boldsymbol{\phi} \times\left(y \boldsymbol{d}_{2}+z \boldsymbol{d}_{3}\right) \cdot \boldsymbol{d}_{1} \\
\boldsymbol{\phi} \times\left(y \boldsymbol{d}_{2}+z \boldsymbol{d}_{3}\right) \cdot \boldsymbol{d}_{2} \\
\boldsymbol{\phi} \times\left(y \boldsymbol{d}_{2}+z \boldsymbol{d}_{3}\right) \cdot \boldsymbol{d}_{3}
\end{array}\right]=\left[\begin{array}{c}
x-y V_{x}-z W_{x} \\
y+V-z \Theta \\
z+W+y \Theta
\end{array}\right]
$$


Differentiating $x_{1}, y_{1}$, and $z_{1}$ with respect to $x$ yields

$$
\left[\begin{array}{c}
x_{1, x} \\
y_{1, x} \\
z_{1, x}
\end{array}\right]=\left[\begin{array}{c}
1-y V_{x x}+z \gamma^{\prime} V_{x}-z W_{x x}-y \gamma^{\prime} W_{x} \\
-z \gamma^{\prime}+V_{x}-z \Theta_{x}-y \gamma^{\prime} \Theta \\
y \gamma^{\prime}+W_{x}+y \Theta_{x}-z \gamma^{\prime} \Theta
\end{array}\right]
$$

Neglecting the transverse shear effect, the longitudinal strain is computed as ${ }^{13}$

$$
\varepsilon=\frac{d s_{1}-d s}{d s}=\frac{s_{1, x}}{s_{x}}-1
$$

where

$$
\begin{gathered}
s_{x}=\sqrt{1+y_{x}^{2}+z_{x}^{2}}=\sqrt{1+\left(y^{2}+z^{2}\right)\left(\gamma^{\prime}\right)^{2}} \\
s_{1, x}=\sqrt{x_{1, x}^{2}+y_{1, x}^{2}+z_{1, x}^{2}} \\
=\sqrt{s_{x}^{2}-2 y V_{x x}-2 z W_{x x}+2\left(y^{2}+z^{2}\right) \gamma^{\prime} \Theta_{x}+\left(x_{1, x}-1\right)^{2}+\left(y_{1, x}+z \gamma^{\prime}\right)^{2}+\left(z_{1, x}-y \gamma^{\prime}\right)^{2}}
\end{gathered}
$$

Ignoring the second-order terms and using the Taylor series expansion, $s_{1, x}$ is approximated as

$$
s_{1, x} \approx s_{x}+\frac{-y V_{x x}-z W_{x x}+\left(y^{2}+z^{2}\right) \gamma^{\prime} \Theta_{x}}{s_{x}}
$$

The longitudinal strain is then obtained as

$$
\begin{aligned}
\varepsilon & =\frac{-y V_{x x}-z W_{x x}+\left(y^{2}+z^{2}\right) \gamma^{\prime} \Theta_{x}}{s_{x}^{2}} \\
& \approx-y\left[1+\left(y^{2}+z^{2}\right)\left(\gamma^{\prime}\right)^{2}\right] V_{x x}-z\left[1+\left(y^{2}+z^{2}\right)\left(\gamma^{\prime}\right)^{2}\right] W_{x x}+\left(y^{2}+z^{2}\right) \gamma^{\prime} y\left[1+\left(y^{2}+z^{2}\right)\left(\gamma^{\prime}\right)^{2}\right] \Theta_{x}
\end{aligned}
$$

For a small wing twist angle $\gamma,\left(\gamma^{\prime}\right)^{2} \approx 0$. Then

$$
\varepsilon=-y V_{x x}-z W_{x x}+\left(y^{2}+z^{2}\right) \gamma^{\prime} \Theta_{x}
$$

The moments acting on the wing are then obtained as ${ }^{13}$

$$
\begin{aligned}
{\left[\begin{array}{c}
M_{x} \\
M_{y} \\
M_{z}
\end{array}\right]=\left[\begin{array}{c}
G J \Theta_{x} \\
0 \\
0
\end{array}\right]+\iint E \varepsilon\left[\begin{array}{c}
\left(y^{2}+z^{2}\right)\left(\gamma^{\prime}+\Theta_{x}\right) \\
-z \\
-y
\end{array}\right] d y d z } \\
=\left[\begin{array}{ccc}
G J+E B_{1}\left(\gamma^{\prime}\right)^{2} & -E B_{2} \gamma^{\prime} & -E B_{3} \gamma^{\prime} \\
-E B_{2} \gamma^{\prime} & E I_{y y} & -E I_{y z} \\
-E B_{3} \gamma^{\prime} & -E I_{y z} & E_{I z z}
\end{array}\right]\left[\begin{array}{c}
\Theta_{x} \\
W_{x x} \\
V_{x x}
\end{array}\right]
\end{aligned}
$$

where $E$ is the Young's modulus; $G$ is the shear modulus; $\gamma^{\prime}$ is the derivative of the wing pre-twist angle; $I_{y y}, I_{y z}$, and $I_{z z}$ are the section area moments of inertia about the flapwise axis; $J$ is the torsional constant; and $B_{1}, B_{2}$, and $B_{3}$ are the bending-torsion coupling constants which are defined as

$$
\left[\begin{array}{l}
B_{1} \\
B_{2} \\
B_{3}
\end{array}\right]=\iint\left(y^{2}+z^{2}\right)\left[\begin{array}{c}
y^{2}+z^{2} \\
z \\
y
\end{array}\right] d y d z
$$

The strain analysis shows that, for a pre-twisted wing, the bending deflections are coupled to the torsional deflection via the slope of the wing pre-twist angle. This coupling can be significant if the wash-out slope $\gamma^{\prime}$ is dominant as in highly twisted wings such as turbomachinery blades. 


\section{Coupled Bending-Torsion Equations}

Without considering chordwise bending of the wing, the equilibrium conditions for bending and torsion are expressed as $^{13}$

$$
\begin{gathered}
\frac{\partial M_{x}}{\partial x}=-m_{x} \\
\frac{\partial^{2} M_{y}}{\partial x}=f_{z}-\frac{\partial m_{y}}{\partial x}
\end{gathered}
$$

where $m_{x}$ is the pitching moment per unit span about the elastic axis, $f_{z}$ is the lift force per unit span, and $m_{y}$ is the bending moment per unit span about the flapwise axis of the wing.

\section{Aerodynamic Forces and Moments}

Because the structural modeling is intended for use in a static aeroelasticity model, a steady-state aerodynamics model is used. Aerodynamic information can be obtained through vortex-lattice modeling to develop the forces and moments for coupled bending-torsion of a flexible wing.

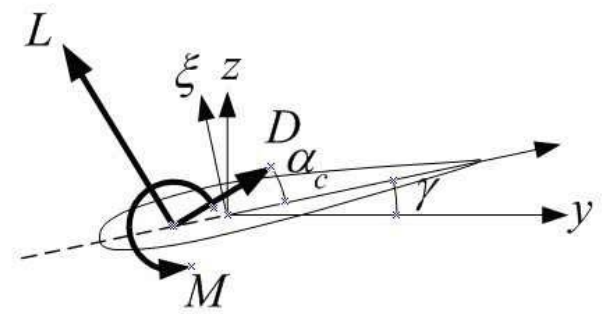

Figure 5. Airfoil Forces and Moments

Neglecting the effect of downwash that is caused due to lift generation over a three-dimensional finite-wing, the sectional lift coefficient for an airfoil cross section, assuming linear aerodynamics, is as follows:

$$
c_{L}(x)=c_{L_{\alpha}}(x) \alpha_{c}(x)
$$

where $\alpha_{c}$ is known as the aeroelastic angle of attack and comprises of the rigid-body angle of attack and the contribution due to aeroelastic deformation. Note that $\alpha_{c}$ is defined relative to the elastic axis of the wing and $c_{L}$ is the sectional lift coefficient in the wing reference frame.

Let $\alpha$ be the aircraft's rigid-body angle of attack and $\alpha_{e}$ be the effect on the local angle of attack due to aeroelastic deformation at the aerodynamic center of the airfoil section. Note that these values $\alpha, \alpha_{e}$ are defined relative to the aircraft pitch axis, not the elastic axis.

$$
\begin{gathered}
\alpha_{c}(x)=\frac{\alpha+\alpha_{e}(x)}{\cos \Lambda} \\
c_{L_{r}}(x)=c_{L_{0}}+c_{L_{\alpha}}(x) \frac{\alpha}{\cos \Lambda} \\
c_{L_{e}}(x)=c_{L_{\alpha}}(x) \frac{\alpha_{e}(x)}{\cos \Lambda}
\end{gathered}
$$

where $c_{L_{0}}$ is the zero angle of attack lift coefficient for the airfoil section.

It is also important to note that the elastic contribution to the local aeroelastic angle of attack, $\alpha_{e}$, can be used to characterize an aeroelastic deformation. Given a deformation characterized by elastic axis twist $\Theta$ and vertical bending slope $W_{x}$, the elastic contribution to the aeroelastic angle of attack can be calculated as

$$
\alpha_{e}(x)=-\Theta(x) \cos \Lambda \cos \Gamma-W_{x}(x) \sin \Lambda
$$

where $\alpha_{e}$ is about the aircraft pitch axis.

The steady-state drag coefficient can be modeled by a parabolic drag polar as

$$
c_{D}(x)=c_{D_{0}}(x)+k(x) c_{L}^{2}(x)
$$


where $c_{D_{0}}$ is the section parasite drag coefficient and $k$ is the section drag polar parameter. This can be expressed in terms of rigid-body and aeroelastic contributions:

$$
\begin{gathered}
c_{D}(x)=c_{D_{r}}(x)+c_{D_{e}}(x) \\
c_{D_{r}}(x)=c_{D_{0}}(x)+k(x) c_{L_{r}}^{2} \\
c_{D_{e}}(x)=k(x) c_{L_{e}}(x)\left[2 c_{L_{r}}(x)+c_{L_{e}}(x)\right]
\end{gathered}
$$

The pitching moment coefficient about the aircraft pitch axis can be computed as

$$
c_{m}(x)=c_{m_{a c}}(x)+\frac{e(x)}{c(x)} c_{L}(x) \cos \Lambda
$$

where $e$ is the location of the aerodynamic center relative to the elastic axis defined in the streamwise direction perpendicular to the pitch axis, positive when the aerodynamic center is forward of the elastic axis, and $c_{m_{a c}}$ is defined about the pitch axis, positive nose-up.

The lift force, drag force, and pitching moment about the aircraft pitch axis are expressed as

$$
\begin{gathered}
l=c_{L} q_{\infty} \cos \Lambda c \\
d=c_{D} q_{\infty} \cos \Lambda c \\
m=c_{m} q_{\infty} c^{2}
\end{gathered}
$$

where $\cos \Lambda$ takes into account the correction due to the elastic axis sweep, but is not needed in the pitch moment calculation since Eq. 27 is already about the pitch axis.

The forces and moments in the local coordinate reference frame are obtained as

$$
\begin{gathered}
f_{x}^{a}=(l \cos \alpha+d \sin \alpha) \Gamma+(d \cos \alpha-l \sin \alpha) \sin \Lambda \\
f_{y}^{a}=(d \cos \alpha-l \sin \alpha) \cos \Lambda \\
f_{z}^{a}=l \cos \alpha+d \sin \alpha-(d \cos \alpha-l \sin \alpha) \sin \Lambda \Gamma \\
m_{x}^{a}=-m \cos \Lambda \\
m_{y}^{a}=m \sin \Lambda \\
m_{z}^{a}=m \cos \Lambda \Gamma
\end{gathered}
$$

For a model with only flapwise bending and torsion considered, the beam deflection analysis is affected only by the terms $f_{z}^{a}, m_{x}^{a}$, and $m_{y}^{a}$. The aerodynamic force and moment terms are thus considered to be

$$
\begin{gathered}
f_{z}^{a} \approx c_{L} q_{\infty} \cos ^{2} \Lambda c \\
m_{x}^{a} \approx-c_{m} q_{\infty} \cos ^{2} \Lambda c^{2} \\
\frac{\partial m_{y}^{a}}{\partial x} \approx \frac{\partial c_{m}}{\partial x} q_{\infty} \sin \Lambda \cos \Lambda c^{2}
\end{gathered}
$$

where an additional $\cos \Lambda$ term is introduced due to the change in direction of $q_{\infty}$ due to sweep.

\section{Propulsive Forces and Moments}

For wing-mounted engines, both the engine mass and thrust must be accounted for in the wing structural analysis. The propulsive force and moment vector are expressed in the reference frame $D$ as

$$
\boldsymbol{f}_{e}=\boldsymbol{\delta}\left(x-x_{e}\right)\left[\begin{array}{lll}
T & 0 & m_{e} g
\end{array}\right]\left[\begin{array}{ccc}
-\sin \Lambda & -\cos \Lambda & \sin \Lambda \Gamma \\
-\cos \Lambda & \sin \Lambda & \cos \Lambda \gamma \\
-\Gamma & 0 & -1
\end{array}\right]\left[\begin{array}{l}
\boldsymbol{d}_{1} \\
\boldsymbol{d}_{2} \\
\boldsymbol{d}_{3}
\end{array}\right]=\delta\left(x-x_{e}\right)\left[\begin{array}{c}
\left(-T \sin \Lambda-m_{e} g \Gamma\right) \boldsymbol{d}_{1} \\
-T \cos \Lambda \boldsymbol{d}_{2} \\
\left(T \sin \Lambda \Gamma-m_{e} g\right) \boldsymbol{d}_{3}
\end{array}\right]
$$




$$
\boldsymbol{m}_{e}=\boldsymbol{r}_{e} \times \boldsymbol{f}_{e}=\left(x_{e} \boldsymbol{d}_{1}-y_{e} \boldsymbol{d}_{2}-z_{e} \boldsymbol{d}_{3}\right) \times \boldsymbol{f}_{e}=\boldsymbol{\delta}\left(x-x_{e}\right)\left[\begin{array}{c}
\left(-T y_{e} \sin \Lambda \Gamma-T z_{e} \cos \Lambda+m_{e} g y_{e}\right) \boldsymbol{d}_{1} \\
\left(-T y_{e} \sin \Lambda \Gamma+m_{e} g x_{e}+T z_{e} \sin \Lambda+m_{e} g y_{e} \Gamma\right) \boldsymbol{d}_{2} \\
\left(-T x_{e} \cos \Lambda \Gamma-T y_{e} \sin \Lambda-m_{e} g y_{e} \Gamma\right) \boldsymbol{d}_{3}
\end{array}\right]
$$

where $T$ is the engine thrust, $m_{e}$ is the engine mass, $\left(x_{e}, y_{e}, z_{e}\right)$ is the coordinate of the engine thrust center such that $y_{e}$ is positive forward of the elastic axis and $z_{e}$ is positive below the elastic axis, and $\delta\left(x-x_{e}\right)$ is the Dirac delta function such that

$$
\int \delta\left(x-x_{e}\right) f(x) d x=f\left(x_{e}\right)
$$

Transforming into the local coordinate reference frame, the propulsive forces and moments are given by

$$
\begin{aligned}
& f_{x}^{e}=\delta\left(x-x_{e}\right)\left[-T \sin \Lambda-m_{e} g \Gamma+\left(T \sin \Lambda \Gamma-m_{e} g\right) W_{x}\right] \\
& f_{y}^{e}=\delta\left(x-x_{e}\right)\left[-T \cos \Lambda+\left(T \sin \Lambda \Gamma-m_{e} g\right)(\Theta+\gamma)\right] \\
& f_{z}^{e}=\delta\left(x-x_{e}\right)\left[T \sin \Lambda \Gamma-m_{e} g+T \cos \Lambda(\Theta+\gamma)+\left(T \sin \Lambda+m_{e} g \Gamma\right) W_{x}\right] \\
& m_{x}^{e}=\delta\left(x-x_{e}\right)\left[-T y_{e} \sin \Lambda \Gamma-T z_{e} \cos \Lambda+m_{e} g y_{e}-\left(T x_{e} \cos \Lambda+T y_{e} \sin \Lambda+m_{e} g y_{e} \Gamma\right) W_{x}\right] \\
& m_{y}^{e}=\delta\left(x-x_{e}\right)\left[-T x_{e} \sin \Lambda \Gamma+m_{e} g x_{e}+T z_{e} \sin \Lambda+m_{e} g z_{e} \Gamma-\left(T x_{e} \cos \Lambda+T y_{e} \sin \Lambda+m_{e} g y_{e} \Gamma\right)(\Theta+\gamma)\right] \\
& m_{z}^{e}=\delta\left(x-x_{e}\right)\left[-T x_{e} \cos \Lambda-T y_{e} \sin \Lambda-m_{e} g y_{e} \Gamma+T x_{e} \sin \Lambda \Gamma-m_{e} g x_{e}-T z_{e} \sin \Lambda-m_{e} g z_{e} \Gamma\right)(\Theta+\gamma) \\
& \left.+\left(T y_{e} \sin \Lambda \Gamma+T z_{e} \cos \Lambda-m_{e} g y_{e}\right) W_{x}\right]
\end{aligned}
$$

The partial derivatives of the moment components are

$$
\begin{gathered}
\frac{\partial m_{x}^{e}}{\partial x}=-\delta\left(x-x_{e}\right)\left(T x_{e} \cos \Lambda+T y_{e} \sin \Lambda+m_{e} g y_{e} \Gamma\right) W_{x x} \\
\frac{\partial m_{y}^{e}}{\partial x}=-\delta\left(x-x_{e}\right)\left(T x_{e} \cos \Lambda+T y_{e} \sin \Lambda+m_{e} g y_{e} \Gamma\right)\left(\Theta_{x}+\gamma^{\prime}\right) \\
\frac{\partial m_{z}^{e}}{\partial x}=- \\
\quad \delta\left(x-x_{e}\right)\left[\left(T x_{e} \sin \Lambda \Gamma-m_{e} g x_{e}-T z_{e} \sin \Lambda-m_{e} g z_{e} \Gamma\right)\left(\Theta_{x}+\gamma^{\prime}\right)\right. \\
\left.+\left(T y_{e} \sin \Lambda \Gamma+T z_{e} \cos \Lambda-m_{e} g y_{e} \Gamma\right) W_{x x}\right]
\end{gathered}
$$

\section{Summary of Coupled Bending-Torsion Partial Differential Equations}

The force and moment equations due to aerodynamics and propulsive sources and inertial effects are formulated.

$$
\begin{gathered}
f_{z}=f_{z}^{a}+f_{z}^{e}-m g-m W_{t t}+m e_{c g} \Theta_{t t}+\delta\left(x-x_{e}\right)\left(-m_{e} W_{t t}+m_{e} y_{e} \Theta_{t t}\right) \\
m_{x}=m_{x}^{a}+m_{x}^{e}+m g e_{c g}+m e_{c g} W_{t t}-m r_{k}^{2} \Theta_{t t}+\delta\left(x-x_{e}\right)\left[m_{e} y_{e} W_{t t}-m_{e}\left(y_{e}^{2}+z_{e}^{2}\right) \Theta_{t t}\right] \\
\frac{\partial m_{y}}{\partial x}=\frac{\partial m_{y}^{a}}{\partial x}+\frac{\partial m_{y}^{e}}{\partial x}
\end{gathered}
$$

Inserting Eq. 13 and the force and moment terms into the governing equilibrium equations, Eqs. 16 and 17, the following equations which describe the coupled bending and torsion motion of the wing can be formulated:

$$
\begin{aligned}
& \frac{\partial^{2}}{\partial x^{2}}\left(-E B_{2} \gamma^{\prime} \Theta_{x}+E I_{y y} W_{x x}\right)= \\
& -m W_{t t}+m e_{c g} \Theta_{t t}-m g+c_{L} q_{\infty} \cos ^{2} \Lambda c-\frac{\partial c_{m}}{\partial x} \tan \Lambda q_{\infty} \cos ^{2} \Lambda c^{2} \\
& +\delta\left(x-x_{e}\right)\left(-m_{e} w_{t t}+m_{e} y_{e} \Theta_{t t}\right) \\
& +\delta\left(x-x_{e}\right)\left[T \sin \Lambda \Gamma-m_{e} g+\left(T \sin \Lambda+m_{e} g \Gamma\right) W_{x}+T \cos \Lambda(\Theta+\gamma)\right] \\
& +\delta\left(x-x_{e}\right)\left(T x_{e} \cos \Lambda+T y_{e} \sin \Lambda+m_{e} g y_{e} \Gamma\right)\left(\Theta_{x}+\gamma^{\prime}\right) \\
& +\delta\left(x-x_{e}\right)\left(-T y_{e} \sin \Lambda \Gamma-T z_{e} \cos \Lambda+m_{e} g y_{e}\right)\left[W_{x x}(\Theta+\gamma)+W_{x}\left(\Theta_{x}+\gamma^{\prime}\right)\right] \\
& \frac{\partial}{\partial x}\left\{\left[G J+E B_{1}\left(\gamma^{\prime}\right)^{2}\right] \Theta_{x}-E B_{2} \gamma^{\prime} W_{x x}\right\}= \\
& m r_{k}^{2} \Theta_{t t}-m e_{c g} W_{t t}-m g e_{c g}+c_{m} q_{\infty} \cos { }^{2} \Lambda c^{2}+\delta\left(x-x_{e}\right)\left[m_{e}\left(y_{e}^{2}+z_{e}^{2}\right) \Theta_{t t}-m_{e} y_{e} W_{t t}\right] \\
& -\delta\left(x-x_{e}\right)\left[-T y_{e} \sin \Lambda \Gamma-T z_{e} \cos \Lambda+m_{e} g y_{e}-\left(T x_{e} \cos \Lambda+T y_{e} \sin \Lambda+m_{e} g y_{e} \Gamma\right) W_{x}\right]
\end{aligned}
$$




\section{Finite-Element Modeling}

The development of the coupled bending-torsion partial differential equations allows for wing bending and torsional deflections to be solved. FEM ${ }^{8}$ a numerical technique that uses locally-defined basis functions to numerically approximate the solution of the governing partial differential equations, is used. The wing structure is discretized into $n$ equally spaced one-dimensional beam elements, and FEM is applied. The bending and torsional deflections can be approximated as

$$
\begin{gathered}
\Theta(x, t)=\sum_{i=1}^{n} \Theta_{i}(x, t) \\
W(x, t)=\sum_{i=1}^{n} W_{i}(x, t)
\end{gathered}
$$

where $i$ refers to the $i$-th element.

For each element, the bending and torsional deflections are approximated as

$$
\begin{aligned}
& \Theta_{i}(x, t)=\psi_{i} \theta_{1_{i}}(t)+\psi_{2}(x) \theta_{2_{i}}(t)=\left[\begin{array}{ll}
\psi_{1}(x) & \psi_{2}(x)
\end{array}\right]\left[\begin{array}{c}
\theta_{1_{i}}(t) \\
\theta_{2_{i}}(t)
\end{array}\right]=N_{\theta}(x) \theta_{i}(t) \\
& W_{i}(x, t)=\left[\phi_{1}(x) w_{1_{i}}(t)+\phi_{2}(x) w_{1_{i}}^{\prime}(t)+\phi_{3}(x) w_{2_{i}}(t)+\phi_{4}(x) w_{2_{i}}^{\prime}(t)\right] \\
& =\left[\begin{array}{llll}
\phi_{1}(x) & \phi_{2}(x) & \phi_{3}(x) & \phi_{4}(x)
\end{array}\right]\left[\begin{array}{c}
w_{1_{i}}(t) \\
w_{1_{i}}^{\prime}(t) \\
w_{2_{i}}(t) \\
w_{2_{i}}^{\prime}(t)
\end{array}\right]=N_{w}(x) w_{i}(t)
\end{aligned}
$$

where the subscripts 1 and 2 denote values at nodes 1 and 2 , and $\psi_{j}(x), j=1,2$ and $\phi_{k}(x), k=1,2,3,4$ are the linear and Hermite polynomial shape functions

$$
\begin{gathered}
\psi_{1}(x)=1-\frac{x}{l} \\
\psi_{2}(x)=\frac{x}{l} \\
\phi_{1}(x)=1-3\left(\frac{x}{l}\right)^{2}+2\left(\frac{x}{l}\right)^{3} \\
\phi_{2}(x)=l\left[\frac{x}{l}-2\left(\frac{x}{l}\right)^{2}+\left(\frac{x}{l}\right)^{3}\right] \\
\phi_{3}(x)=3\left(\frac{x}{l}\right)^{2}-2\left(\frac{x}{l}\right)^{3} \\
\phi_{4}(x)=l\left[-\left(\frac{x}{l}\right)^{2}+\left(\frac{x}{l}\right)^{3}\right]
\end{gathered}
$$

where $x \in[0,1]$ is the local coordinate and $l=\frac{L}{n}$ is the element length.

The weak-form integral expressions of the coupled bending-torsion partial differential equations are obtained by multiplying the equations by $N_{\theta}^{\mathrm{T}}(x)$ and $N_{w}^{\mathrm{T}}(x)$ and then integrating over the wing span. This yields

$$
\begin{aligned}
& \sum_{i=0}^{n} \int_{0}^{l} N_{\theta}^{\mathrm{T}} \frac{d}{d x}\left\{\left[G J+E B_{1}\left(\gamma^{\prime}\right)^{2}\right] N_{\theta}^{\prime} \theta_{i}-E B_{2} \gamma^{\prime} N_{w}^{\prime \prime} w_{i}\right\} d x= \\
& \sum_{i=1}^{n} \int_{0}^{l} N_{\theta}^{\mathrm{T}}\left(c_{m} c\right) q_{\infty} \cos ^{2} \Lambda c d x+\sum_{i=1}^{n} \int_{0}^{l} N_{\theta}^{\mathrm{T}}\left(-m g e_{c g}+m r_{k}^{2} N_{\theta} \ddot{\theta}_{i}-m e_{c g} N_{w} \ddot{w}_{i}\right) d x \\
& +\sum_{i=1}^{n} N_{\theta}^{\mathrm{T}}\left[m_{e}\left(y_{e}^{2}+z_{e}^{2}\right) N_{\theta} \ddot{\theta}_{i}-m_{e} y_{e} N_{w} \ddot{w}_{i}\right]_{x=x_{e}}+ \\
& \sum_{i=1}^{n} N_{\theta}^{\mathrm{T}}\left[T y_{e} \sin \Lambda \Gamma+T z_{e} \cos \Lambda-m_{e} g y_{e}+\left(T x_{e} \cos \Lambda+T y_{e} \sin \Lambda+m_{e} g y_{e} \Gamma\right) N_{w}^{\prime} w_{i}\right] x_{x=x_{e}}
\end{aligned}
$$




$$
\begin{aligned}
& \sum_{i=0}^{n} \int_{0}^{l} N_{w}^{\mathrm{T}} \frac{d^{2}}{d x^{2}}\left(-E B_{2} \gamma^{\prime} N_{\theta}^{\prime} \theta_{i}+E I_{y y} N_{w}^{\prime \prime} w_{i}\right) d x= \\
& \quad \sum_{i=1}^{n} \int_{0}^{l} N_{w}^{\mathrm{T}}\left(c_{L}-\frac{d c_{m}}{d x} \tan \Lambda c\right) q_{\infty} \cos ^{2} \Lambda c d x+\sum_{i=1}^{n} N_{w}^{\mathrm{T}}\left(-\rho g A-\rho A N_{w} \ddot{w}_{i}+\rho A e_{c g} N_{\theta} \ddot{\theta}_{i}\right) d x \\
& \quad+\sum_{i=1}^{n} N_{w}^{\mathrm{T}}\left[-m_{e} N_{w} \ddot{w}_{i}+m_{e} y_{e} N_{\theta} \ddot{\theta}_{i}+T \sin \Lambda \Gamma-m_{e} g+T \cos \Lambda\left(N_{\theta} \theta_{i}+\gamma\right)+\left(T \sin \Lambda+m_{e} g \Gamma\right) N_{w}^{\prime} w_{i}\right]_{x=x_{e}} \\
& \quad+\sum_{i=1}^{n} N_{w}^{\mathrm{T}}\left[\left(T x_{e} \cos \Lambda+T y_{e} \sin \Lambda+m_{e} g y_{e} \Gamma\right)\left(N_{\theta}^{\prime} \theta_{i}+\gamma^{\prime}\right)\right]_{x=x_{e}}
\end{aligned}
$$

The expressions on the left hand sides can be integrated by parts upon enforcing the boundary conditions resulting in

$$
\begin{gathered}
\int_{0}^{l} N_{\theta}^{\mathrm{T}} \frac{d}{d x}\left\{\left[G J+E B_{1}\left(\gamma^{\prime}\right)^{2}\right] N_{\theta}^{\prime} \theta_{i}-E B_{2} \gamma^{\prime} N_{w}^{\prime \prime} w_{i}\right\} d x=-\int_{0}^{l} N_{\theta}^{\prime} \mathrm{T}\left\{\left[G J+E B_{1}\left(\gamma^{\prime}\right)^{2}\right] N_{\theta}^{\prime} \theta_{i}-E B_{2} \gamma^{\prime} N_{w}^{\prime \prime} w_{i}\right\} d x \\
\int_{0}^{l} N_{w}^{\mathrm{T}} \frac{d^{2}}{d x^{2}}\left(-E B_{2} \gamma^{\prime} N_{\theta}^{\prime} \theta_{i}+E I_{y y} N_{w}^{\prime \prime} w_{i}\right) d x=\int_{0}^{l} N_{w}^{\prime \prime T}\left(-E B_{2} \gamma^{\prime} N_{\theta}^{\prime} \theta_{i}+E I_{y y} N_{w}^{\prime \prime} w_{i}\right) d x
\end{gathered}
$$

The elemental mass matrix, stiffness matrix, and force vector are then established as

$$
\begin{aligned}
& M_{i}=\int_{0}^{l} m\left[\begin{array}{cc}
r_{k}^{2} N_{\theta}^{\mathrm{T}} N_{\theta} & -e_{c g} N_{\theta}^{\mathrm{T}} N_{w} \\
-e_{c g} N_{w}^{\mathrm{T}} N_{\theta} & N_{w}^{\mathrm{T}} N_{w}
\end{array}\right] d x+m_{e}\left[\begin{array}{cc}
\left(y_{e}^{2}+z_{e}^{2}\right) N_{\theta}^{\mathrm{T}} N_{\theta} & -y_{e} N_{\theta}^{\mathrm{T}} N_{w} \\
-y_{e} N_{w}^{\mathrm{T}} N_{\theta} & N_{w}^{\mathrm{T}} N_{w}
\end{array}\right]_{x=x_{e}} \\
& K_{i}=\int_{0}^{l}\left[\begin{array}{cc}
\left.G J+E B_{1}\left(\gamma^{\prime}\right)^{2}\right] N_{\theta}^{\prime \mathrm{T}} N_{\theta}^{\prime} & -E B_{2} \gamma^{\prime} N_{\theta}^{\prime} \mathrm{T} N_{w}^{\prime \prime} \\
-E B_{2} \gamma^{\prime} N_{w}^{\prime \prime} N_{\theta}^{\prime} & E I_{y y} N_{w}^{\prime \prime T} N_{w}^{\prime \prime}
\end{array}\right] d x \\
&+\left[\begin{array}{c}
\left.-T x_{e} \cos \Lambda+T y_{e} \sin \Lambda+m_{e} g y_{e} \Gamma\right) N_{\theta}^{\mathrm{T}} N_{w}^{\prime} \\
-T \cos \Lambda N_{w}^{\mathrm{T}} N_{\theta}-\left(T x_{e} \cos \Lambda+T y_{e} \sin \Lambda+m_{e} g y_{e} \Gamma\right) N_{w}^{\mathrm{T}} N_{\theta}^{\prime} \\
-\left(T \sin \Lambda+m_{e} g \Gamma\right) N_{w}^{\mathrm{T}} N_{w}^{\prime}
\end{array}\right]_{x=x_{e}} \\
& F_{i}=\int_{0}^{l} m g\left[\begin{array}{c}
e_{c g} N_{\theta}^{\mathrm{T}} \\
-N_{w}^{T}
\end{array}\right] d x+\int_{0}^{l}\left(\left[\begin{array}{c}
-c_{m} c N_{\theta}^{\mathrm{T}} \\
c_{L} N_{w}^{T}
\end{array}\right]+\left[\begin{array}{c}
0 \\
c_{m} c \tan \Lambda N_{w}^{\prime T}
\end{array}\right]\right) q_{\infty} \cos ^{2} \Lambda c d x \\
&+\left[\begin{array}{c}
0 \\
c_{m} c \tan \Lambda N_{w}^{T}
\end{array}\right]_{0}^{l} q_{\infty} \cos { }^{2} \Lambda c d x \\
&+\left[\begin{array}{c}
{\left[\sin \Lambda \Gamma-m_{e} g+T \cos \Lambda \gamma+\left(T x_{e} \cos \Lambda+T y_{e} \sin \Lambda+m_{e} g y_{e} \Gamma\right) \gamma^{\prime}\right] N_{w}^{T}}
\end{array}\right]_{x=x_{e}}
\end{aligned}
$$

The globally assembled system is described by the matrix equation

$$
M \ddot{x}_{e}+K x_{e}=F
$$

where $x_{e}=\left[\begin{array}{lllllllllllllll}\theta_{1} & w_{1} & w_{1}^{\prime} & \theta_{2} & w_{2} & w_{2}^{\prime} & \ldots & \theta_{i} & w_{i} & w_{i}^{\prime} & \ldots & \theta_{n+1} & w_{n+1} & w_{n+1}^{\prime}\end{array}\right]^{\mathrm{T}}$.

Equation 74 represents the governing equation for solving the structural deflection of a flexible wing given aerodynamic and propulsive force and moment inputs. By setting $\ddot{x}_{e}=\mathbf{0}$, the equilibrium solution can be obtained through inverting the stiffness matrix and pre-multiplying the force matrix. This represents the static structural deflection of the wing based on the prescribed load input at that flight condition.

$$
x_{e}=K^{-1} F
$$

Information extracted from the wing solution state vector $x_{e}$ is used to characterize the wing's structural deflection along the elastic axis of the wing $\Theta$ and $W$. It can also be used to calculate the elastic contribution to the aeroelastic angle of attack $\alpha_{e}$ in Eq. 22. 


\section{Vortex-Lattice Aerodynamic Modeling}

Vorview is a computational tool used for aerodynamic modeling of aircraft configurations using vortex-lattice method. ${ }^{14}$ Based on lifting line aerodynamic theory, Vorview provides a rapid method for estimating aerodynamic force and moment coefficients. Input vehicle geometries are discretized within Vorview into a series of panels which are then represented by placement of spanwise and chordwise locations of bound or horseshoe vortices. Vorview computes the vehicle aerodynamics in both the longitudinal and lateral directions independently, and these can be combined to produce the overall aerodynamic characteristics of the vehicle at any arbitrary angle of attack and angle of sideslip.

Vorview is considered a medium fidelity tool, and limitations associated with vortex-lattice modeling in general apply to Vorview aerodynamic analysis. The drag prediction by Vorview is most reliable only for induced drag prediction due to the inviscid nature of any vortex-lattice method. Prediction of viscous drag due to boundary layer separation and wave drag due to shock-induced boundary layer separation are generally not conducted by vortexlattice, and viscous drag must be estimated using other methods.

In addition to force and moment analysis, Vorview can provide a rapid estimation of aerodynamic derivatives including dynamic derivatives due to angular rates. These aerodynamic stability and control derivatives are useful in analyzing the stability and handling characteristics of an aircraft configuration. Owing to the computationally efficient vortex-lattice method, aerodynamic derivatives can be estimated in Vorview fairly quickly. A flight dynamic model for a given vehicle configuration can be easily developed with Vorview, using the results from these stability and handling analyses. Vorview has been validated by both wind tunnel data ${ }^{10}$ as well as the NASA Cart3D tool, ${ }^{15}$ which is a high-fidelity inviscid (Euler) CFD analysis code targeted at analyzing aircraft performance in conceptual and preliminary aerodynamic design. In general, both Vorview and Cart3D seem to have similar predictive capabilities when compressibility is not a factor.

Figure 6 illustrates an aerodynamic model of the GTM in Vorview.

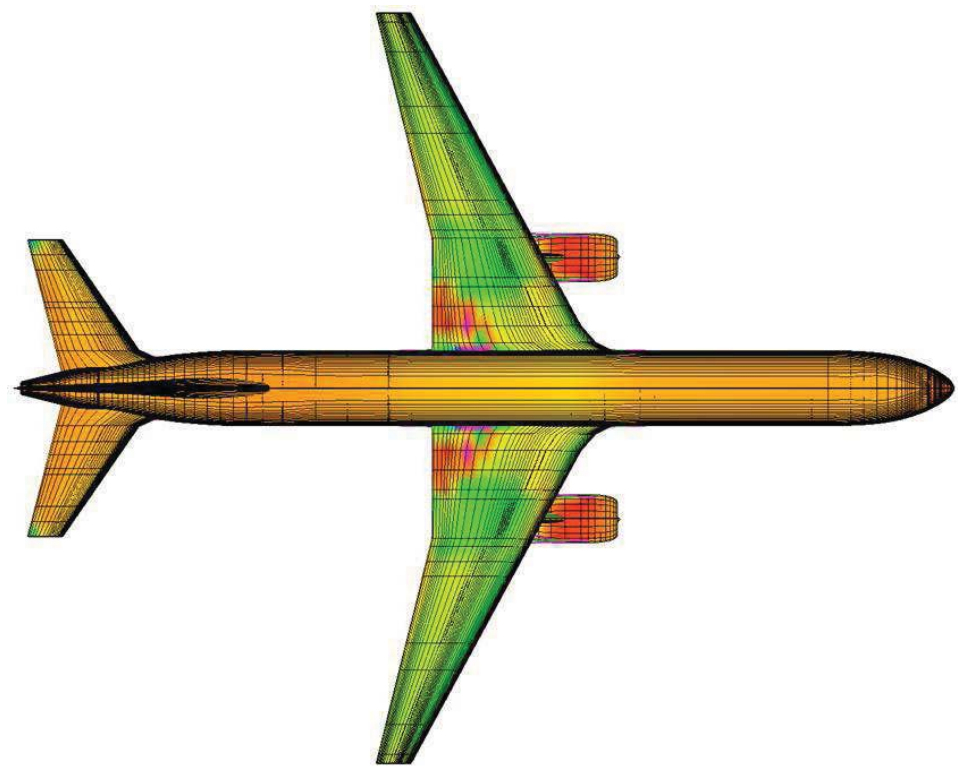

Figure 6. GTM Aircraft Model in Vorview

In this study, Vorview will be used as the primary tool for conducting aerodynamic modeling for the aircraft configurations. Total aircraft characteristics as well as sectional data along the aircraft wing surfaces can be postprocessed from Vorview.

\section{Parasitic Drag Modeling}

Due to the inviscid nature of vortex-lattice drag estimates, a conceptual parasitic drag model will be utilized for development of the longitudinal trim model. This is necessary for realistic trim thrust values to be calculated, which must take into account viscous drag over the ESAC model's airframe. 
The skin friction model for all the surfaces will be done by calculating the arc lengths of the surfaces along the direction of flow and treating those lengths as flat plate surfaces. For airfoil surfaces, the arc lengths can be divided into top and bottom arc surfaces. For revolved surfaces, the model will calculate the skin friction coefficients by revolving the mean arc lengths.

The Reynold's number for an arc length $c$ is calculated as

$$
R e_{c}=\frac{\rho V c}{\mu}
$$

A critical Reynold's number $R e_{x_{c}}$ is assumed to be $R e_{x_{c}}=600,000$. This critical Reynold's number is used to calculate the critical length as

$$
x_{c}=\frac{\operatorname{Re}_{x_{c}} \mu}{\rho V}
$$

The kinematic viscosity model is calculated in $\frac{\mathrm{lb}-\mathrm{s}}{\mathrm{ft}^{2}}$ by the Sutherland's viscosity model:

$$
\mu=0.3170 \times 10^{-10} T^{\frac{3}{2}}\left(\frac{734.7}{T+216}\right)
$$

The revised skin friction coefficient for a single arc length is calculated as

$$
c_{f}=\underbrace{\frac{1.328}{\sqrt{\operatorname{Re}_{x_{c}}}} \frac{x_{c}}{c}}_{c_{f, \text { laminar }}}+\underbrace{0.072 R e_{c}^{-0.2}-0.072 \frac{x_{c}}{c} R e_{x_{c}}^{-0.2}}_{c_{f, \text { turbulent }}}
$$

The total skin friction coefficient for the aircraft components can then be formulated by integrating the skin friction coefficients either over the entire span of a wing, or the entire revolution of a surface.

If pressure drag is to be conceptually estimated as well, then a form factor for the wing needs to be estimated. A form factor $F F$ can can be defined for a wing surface as ${ }^{16}$

$$
F F=\left(1+\frac{0.6}{(x / c)_{m}}\left(\frac{t}{c}\right)+100\left(\frac{t}{c}\right)^{4}\right)\left(1.34 M^{0.18}\left(\cos \Lambda_{m}\right)^{0.28}\right)
$$

where $\frac{t}{c}$ is the maximum thickness of the airfoil, $\left(\frac{x}{c}\right)_{m}$ is the location of the airfoil maximum thickness point, and $\Lambda_{m}$ is the sweep of the maximum-thickness line.

For bodies of revolution, the form factor is evaluated as ${ }^{16}$

$$
F F=\left(1+\frac{60}{f^{3}}+\frac{f}{400}\right)
$$

where $f$ is a fineness ratio given by $f=\frac{l}{\sqrt{\frac{4}{\pi} A_{\max }}}$ and $A_{\max }$ is the largest cross-sectional area perpendicular to the flow.

The total parasitic drag coefficient including skin-friction and pressure drag due to separation and the supervelocity effect is calculated as ${ }^{16}$

$$
C_{D_{0}}=\sum \frac{C_{f}(F F)(Q) S_{\text {wet }}}{S_{\text {ref }}}
$$

where all the components' contributions to parasitic drag are summed up. The value $S_{\text {wet }}$ represents the wetted surface area of the component, and $Q$ represents an interference factor.

The parasite drag modeling method introduced here is also used to develop the wing's spanwise parasitic drag coefficients used in Eq. 23 of the aeroelastic model.

\section{Automated Geometry Modeling Tool}

An automated geometry generation tool is developed in Matlab and is used to close the loop between the structural and aerodynamic modeling needed to generate an aeroelastic model. The geometry generation tool uses structural deflection data that is computed by the FEM model and applies it to the undeformed aircraft wing geometry to reflect static aeroelastic deflections. The vehicle geometry modeler directly outputs a geometry input file that can be read by Vorview when computing an aeroelastic solution. 


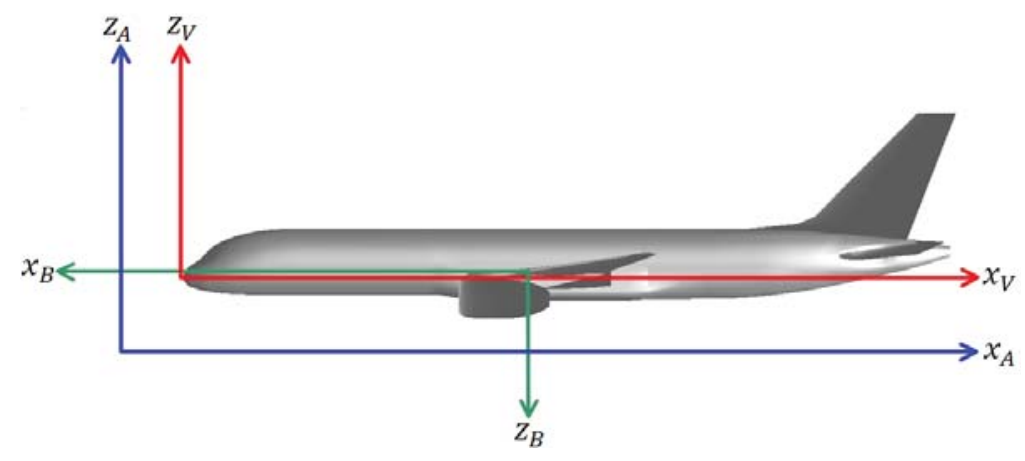

Figure 7. GTM Coordinate Systems

Consider the reference frames in Fig. 7. The coordinate reference frame $\left(x_{A}, y_{A}, z_{A}\right)$ defines the Body Station (BS), the Body Butt Line (BBL), and the Body Water Line (BWL) of the aircraft, respectively. The coordinate reference frame $\left(x_{V}, y_{V}, z_{V}\right)$ is the translated coordinate system attached to the nose of the aircraft such that $x_{V}=x_{B}-13.25 \mathrm{ft}$, $y_{V}=y_{B}$, and $z_{V}=z_{B}-15.8333 \mathrm{ft}$. This reference frame is used by the vortex-lattice aerodynamic modeling tool. The aircraft body reference frame $\left(x_{B}, y_{B}, z_{B}\right)$ is the same $B$ coordinate system defined earlier in Fig. 3 by the unit vectors $\boldsymbol{b}_{1}, \boldsymbol{b}_{2}$, and $\boldsymbol{b}_{3}$. The $B$ coordinate frame is attached to the aircraft center of gravity (CG) such that $x_{B}=\bar{x}_{V}-x_{V}$, $y_{B}=y_{V}-\bar{y}_{V}$, and $z_{B}=\bar{z}_{V}-z_{V}$, where $\left(\bar{x}_{V}, \bar{y}_{V}, \bar{z}_{V}\right)$ is the coordinate of the CG in the $\left(x_{V}, y_{V}, z_{V}\right)$ reference frame. ${ }^{11}$

The vehicle geometry modeler has access to the outer mold line of the aircraft geometry. It is capable of applying geometric transformations onto the outer mold coordinates of the wing's jig-shape to simulate aeroelastic deflection. Neglecting chordwise bending deflection and utilizing the coordinate system of the left wing developed earlier (coordinate frame $D$ ), the aeroelastic deflections in bending and torsion are expressed in a vector form as

$$
\begin{gathered}
\boldsymbol{\phi}=\Theta \boldsymbol{d}_{1}-W_{x} \boldsymbol{d}_{2} \\
\Delta \boldsymbol{r}=-W \sin W_{x} \boldsymbol{d}_{1}+W \cos W_{x} \boldsymbol{d}_{3}
\end{gathered}
$$

The coordinate reference frame $(x, y, z)$ of the left wing is related to the coordinate reference frame $\left(x_{V}, y_{V}, z_{V}\right)$ by the relationship

$$
\begin{aligned}
{\left[\begin{array}{l}
\boldsymbol{d}_{1} \\
\boldsymbol{d}_{2} \\
\boldsymbol{d}_{3}
\end{array}\right]=} & {\left[\begin{array}{ccc}
-\sin \Lambda \cos \Gamma & -\cos \Lambda \sin \Gamma & -\sin \Gamma \\
-\cos \Lambda & \sin \Lambda & 0 \\
\sin \Lambda \sin \gamma & \cos \Lambda \sin \Gamma & -\cos \Gamma
\end{array}\right]\left[\begin{array}{l}
\boldsymbol{b}_{1} \\
\boldsymbol{b}_{2} \\
\boldsymbol{b}_{3}
\end{array}\right] } \\
= & {\left[\begin{array}{ccc}
-\sin \Lambda \cos \Gamma & -\cos \Lambda \cos \Gamma & -\sin \Gamma \\
-\cos \Lambda & \sin \Lambda & 0 \\
\sin \Lambda \sin \Gamma & \cos \Lambda \sin \Gamma & -\cos \Gamma
\end{array}\right]\left[\begin{array}{c}
-\boldsymbol{v}_{1} \\
\boldsymbol{v}_{2} \\
-\boldsymbol{v}_{3}
\end{array}\right] }
\end{aligned}
$$

where $\left(\boldsymbol{v}_{1}, \boldsymbol{v}_{2}, \boldsymbol{v}_{3}\right)$ are the unit vectors for the Vorview coordinate reference frame $\left(x_{V}, y_{V}, z_{V}\right)$.

Thus, the aeroelastic deflections result in a wing twist expressed as an incremental angle of attack $\Delta \alpha$ (positive nose-up), a horizontal deflection $\Delta y_{V}$ (positive deflection towards wing tip), and a vertical deflection $\Delta z_{V}$ (positive displacement upward):

$$
\begin{gathered}
\Delta \alpha=-\Theta \cos \Lambda \cos \Gamma-W_{x} \sin \Lambda \\
\Delta y_{V}=-W \sin W_{x} \cos \Lambda \cos \Gamma-W \cos W_{x} \cos \Lambda \sin \Gamma \\
\Delta z_{v}=-W \sin W_{x} \sin \Gamma+W \cos W_{x} \cos \Gamma
\end{gathered}
$$

A coordinate transformation to account for wing aeroelastic deflections is performed by rotating a wing section about its elastic axis by the incremental angle of attack $\Delta \alpha$ and then translating the resultant coordinates by the horizontal deflection $\Delta y_{V}$ and the vertical deflection $\Delta z_{V}$.

Note that the value of $\Delta \alpha$ is equivalent to the value of $\alpha_{e}$, or the local change in the angle of attack for a wing section due to aeroelastic deformation represented by Eq. 22 . 


\section{Static Aeroelastic Model}

In a standard static aeroelastic model, it is understood that the modeling effort must take into account that aeroelastic structural deformations will affect aircraft aerodynamics, while changing the aerodynamics will thus change the structural deformations. In development of an aeroelastic model, it is crucial to include coupling between a structural and aerodynamic model. Previous studies have analytically constructed fully coupled aeroelastic finite-element models that utilize rigid wing lift-curve slopes as an aerodynamic model. ${ }^{4,5}$

For this study, a static aeroelastic model is developed by closing the loop between a structural FEM model and a vortex-lattice aerodynamic model. For a model involving only flapwise bending and torsion, the aeroelastic deflection can be summarized by the quantities of aeroelastic elastic axis twist $\bar{\Theta}(x)$, aeroelastic vertical (flapwise) bending $\bar{W}(x)$, and aeroelastic vertical bending slope $\bar{W}_{x}(x)$. These quantities are emphasized to be aeroelastic deflections, while the terms $\Theta(x), W(x), W_{x}(x)$ are considered structural deflection terms, which may or may not be the aeroelastic solution for a given flight condition. By closing a static aeroelastic loop as shown in Fig. 8, the structural deflections $\Theta(x)$, $W(x), W_{x}(x)$ are expected to converge to $\bar{\Theta}(x), \bar{W}(x), \bar{W}_{x}(x)$ as iterations are conducted. The structural and aeroelastic deformations can also be represented by the elastic contribution to the aeroelastic angle of attack in Eq. 22, or $\alpha_{e}(x)$ and $\bar{\alpha}_{e}(x)$.

The static aeroelastic code maps an input flight condition corresponding to an angle of attack $\alpha$, Mach number $M$, engine thrust value $T$, and altitude $h$ into the respective static aeroelastic deflection solution a flexible wing would experience at that flight condition. The following procedure is followed:

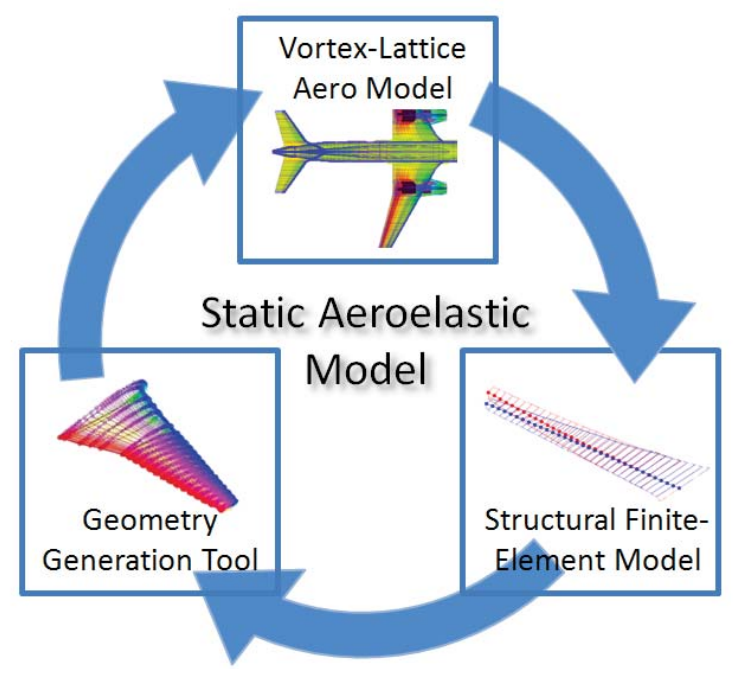

Figure 8. Static Aeroelastic Model Concept

1. Vortex-lattice modeling is conducted on an input geometry at the specified flight condition to determine the aircraft total aerodynamic quantities, as well as sectional coefficients $c_{L}(x), c_{m_{a c}}(x), k(x), c_{L_{\alpha}}(x)$, and $x_{a c}(x)$ or the location of the section aerodynamic centers.

2. The structural FEM model uses the sectional aerodynamic load inputs to calculate the wing's structural deflection $\Theta(x)$ and $W(x)$.

3. The geometry generation tool converts $\Theta(x)$ and $W(x)$ into the series of deformations $\alpha_{e}(x), \Delta y_{V}(x)$, and $\Delta z_{V}(x)$, and generates a new aircraft geometry with the deformed wing.

4. Steps 1-3 are repeated until a convergence criteria is met.

This static aeroelastic model outputs $\bar{\Theta}(x), \bar{W}(x), \bar{W}_{x}(x)$, and $\bar{\alpha}_{e}(x)$, and vortex-lattice can be used to determine the total aircraft coefficients such as $C_{L}, C_{D}$, and $C_{m}$ for the model with aeroelastic deflection. The aeroelastic convergence criteria is placed such that the aircraft $C_{L}$ is evaluated at each iteration and the loop is exited if $\left|\Delta C_{L}\right|$ between iterations is below a certain tolerance. 


\section{Longitudinal Trim Model}

The condition known as "trim" refers to the condition where an aircraft is in a state of constant velocity equilibrium. For longitudinal trim, this formulates a three degree-of-freedom system where the input variables are the aircraft's angle of attack $\alpha$, the elevator deflection $\delta_{e}$ (positive deflection is downward deflection), and the engine thrust $T$.

The forces and moments are developed in the aircraft stability axes shown in Fig. 9. Only symmetric flight within a vertical plane of a non-rotating flat Earth is considered.
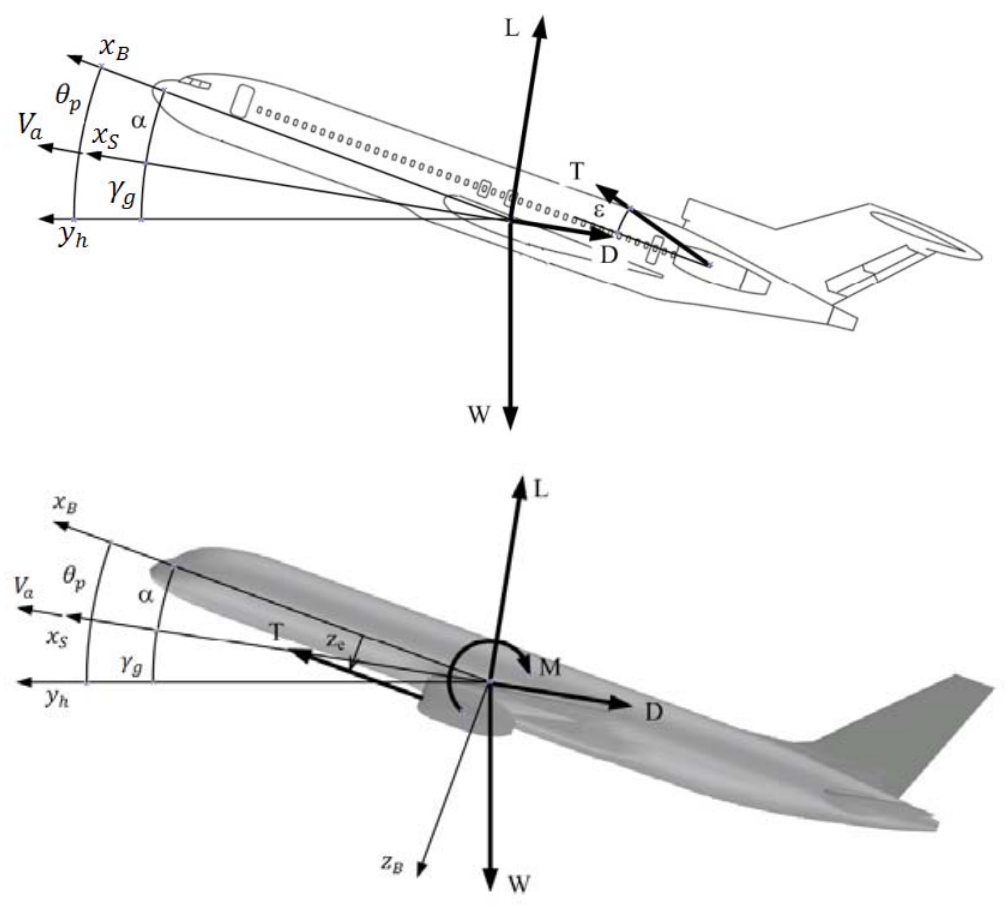

Figure 9. Aircraft Longitudinal Forces and Axes

The body-fixed coordinate system is consistent with the previous usages of the $B$ coordinate frame, while the stability or wind axes are represented by $\left(x_{S}, y_{S}, z_{S}\right)$ where $x_{S}$ is in the direction of $V_{a}$, the aircraft velocity. The axis $y_{h}$ represents a vector parallel to the flat Earth surface and perpendicular to the aircraft vertical altitude. The value $\gamma_{g}$ represents the aircraft glide path angle, and the pitch angle $\theta_{p}$ is defined as the angle between the aircraft forward body axis and the horizontal $y_{h}$ direction. The aircraft is modeled to experience a lift force $L, \operatorname{drag}$ force $D$, weight $W$, and pitching moment $M$ acting at the aircraft CG. The equilibrium equations in the stability axes are:

$$
\begin{aligned}
& \sum F_{x_{S}}=2 T \cos (\alpha+\varepsilon)-D-W \sin (\gamma)=0 \\
& \sum F_{z_{S}}=2 T \sin (\alpha+\varepsilon)+L-W \cos (\gamma)=0 \\
& \sum M_{y_{S}}=M+2 T z_{e} \cos \varepsilon+2 T x_{e} \sin \varepsilon=0
\end{aligned}
$$

where the value $T$ represents a single engine's thrust value and hence a factor of 2 appears in the equations of motion. The value $\varepsilon$ is the engine mount angle, positive upwards.

The equations are expanded using non-dimensional coefficients:

$$
\begin{aligned}
& f_{x_{S}}=2 T \cos (\alpha+\varepsilon)-\left(C_{D_{r}}+C_{D_{e}}+C_{D_{\delta_{e}}} \delta_{e}\right) q_{\infty} S_{r e f}-W \sin \gamma_{g}=0 \\
& f_{z_{S}}=2 T \sin (\alpha+\varepsilon)+\left(C_{L_{r}}+C_{L_{e}}+C_{L_{\delta_{e}}} \delta_{e}\right) q_{\infty} S_{r e f}-W \cos \gamma_{g}=0 \\
& m_{y_{S}}=\left(C_{m_{r}}+C_{m_{e}}+C_{m_{\delta_{e}}} \delta_{e}\right) q_{\infty} S_{r e f} \bar{c}+2 T z_{e} \cos \varepsilon+2 T x_{e} \sin \varepsilon=0
\end{aligned}
$$

Let the static aeroelastic deformation be represented by $\bar{\alpha}_{e}=\bar{\alpha}_{e}\left(y_{S}, \alpha, T\right)$, a function of the wing spanwise station $y_{S}$, the aircraft angle of attack $\alpha$, and the engine thrust $T$. For a model with wing flexibility, it must be taken into 
account that the wing's aeroelastic deformation will change the direction of the thrust vector. Let the coordinates $\left(x_{e_{S}}, y_{e_{S}}, z_{e_{S}}\right)$ represent the location of the engine thrust center in the aircraft stability axes such that $y_{e_{S}}$ represents the location of the engine thrust center along the spanwise axis, $x_{e_{s}}$ represents the distance of the engine thrust center in front of the aircraft center of gravity, and $z_{e_{S}}$ represents the distance of the engine thrust center below the aircraft center of gravity. The equations can then be expanded as

$$
\begin{aligned}
& f_{x_{S}}=2 T \cos \left(\alpha+\varepsilon+\bar{\alpha}_{e}\left(y_{e_{S}}\right)\right)-(\underbrace{C_{D_{0}}+K\left(C_{L_{0}}+C_{L_{\alpha}} \alpha\right)^{2}}_{C_{D_{r}}} \\
& \underbrace{+2 K\left(C_{L_{0}}+C_{L_{\alpha}} \alpha\right) \int C_{L_{\alpha}} \bar{\alpha}_{e} d y_{S}+K\left(\int C_{L_{\alpha}} \bar{\alpha}_{e} d y_{S}\right)^{2}}_{C_{D_{e}}}+C_{D_{\delta_{e}}} \delta_{e}) q_{\infty} S_{r e f}-W \sin \gamma_{g} \\
& f_{z_{S}}=2 T \sin \left(\alpha+\varepsilon+\bar{\alpha}_{e}\left(y_{e_{S}}\right)\right)+(\underbrace{C_{L_{0}}+C_{L_{\alpha}} \alpha}_{C_{L_{r}}}+\underbrace{\int C_{L_{\alpha}} \bar{\alpha}_{e} d y_{S}}_{C_{L_{e}}}+C_{L_{\delta_{e}}} \delta_{e}) q_{\infty} S_{r e f}-W \cos \gamma_{g} \\
& m_{y_{S}}=(\underbrace{C_{m_{0}}+C_{m_{\alpha}} \alpha}_{C_{m_{r}}}+\underbrace{\int C_{m_{\alpha}} \bar{\alpha}_{e} d y_{S}}_{C_{m_{e}}}+C_{m_{\delta_{e}}} \delta_{e}) q_{\infty} S_{r e f} \bar{c}+2 T z_{e} \cos \left(\varepsilon+\bar{\alpha}_{e}\left(y_{e_{S}}\right)\right)+2 T x_{e} \sin \left(\varepsilon+\bar{\alpha}_{e}\left(y_{e_{S}}\right)\right)
\end{aligned}
$$

Newton's method will be used in an iterative fashion to solve the nonlinear system of equations. Newton's method is well-established as one of the standard ways in which nonlinear systems of equations are solved and is dependent on calculation or approximation of the Jacobian for the system of equations. When the Jacobian is well-formulated, Newton's method has a quadratic rate of convergence. ${ }^{17}$ The basic equation for Newton's method for a system of equations where the solution vector is $x_{t}$ is given by

$$
x_{t}^{k+1}=x_{t}^{k}-\left[J\left(x_{t}^{k}\right)\right]^{-1} f\left(x_{t}^{k}\right)
$$

When applied to the trim system, the solution state is given by $x_{t}=\left\{\begin{array}{lll}\alpha & \delta_{e} & T\end{array}\right\}^{\mathrm{T}}$. The Jacobian of the system of equations is represented as $J, f$ is a vector $f=\left\{\begin{array}{lll}f_{x_{S}} & f_{z_{S}} & m_{y_{S}}\end{array}\right\}^{\mathrm{T}}$ representing the values of the nonlinear system of equations, $x_{t}^{k}$ represents the $k$-th iterative solution, $f\left(x_{t}^{k}\right)$ represents the values of the nonlinear system of equations at the $k$-th state, and $\left[J\left(x_{t}^{k}\right)\right]$ represents the Jacobian matrix evaluated at the $k$-th state. Through iteration, the values of $x_{t}^{k+1}$ subsequently approach the solution such that $f=\left\{\begin{array}{lll}0 & 0 & 0\end{array}\right\}$ and the solution vector approaches the trim solution $\bar{x}_{t}$ defined by $\bar{\alpha}, \bar{\delta}_{e}$, and $\bar{T}$.

Taking the partial derivatives of the aircraft longitudinal equations of motion allows us to populate the terms in a Jacobian matrix:

$$
\begin{gathered}
J_{11}=\frac{\delta f_{x_{S}}}{\delta \alpha}=-2 T \sin \left(\alpha+\varepsilon+\bar{\alpha}_{e}\left(y_{e_{S}}\right)\right)\left(1+\frac{\partial \bar{\alpha}_{e}\left(y_{e_{S}}\right)}{\partial \alpha}\right) \\
-\left(2 K C_{L_{\alpha}}^{2} \alpha+2 K C_{L_{\alpha}} \int C_{L_{\alpha}} \bar{\alpha}_{e} d y_{S}+2 K\left(C_{L_{0}}+C_{L_{\alpha}} \alpha+1\right) \frac{\partial \int C_{L_{\alpha}} \bar{\alpha}_{e} d y_{S}}{\partial \alpha}\right) q_{\infty} S_{r e f} \\
J_{12}=\frac{\partial f_{x_{S}}}{\partial \delta_{e}}=-C_{D_{\delta_{e}}} q_{\infty} S_{r e f} \\
-\left(2 K\left(C_{L_{0}}+C_{L_{\alpha}} \alpha+1\right) \frac{\partial \int C_{L_{\alpha}} \bar{\alpha}_{e} d y_{S}}{\partial T}\right) q_{\infty} S_{r e f} \\
J_{13}=\frac{\partial f_{x_{S}}}{\partial T}=2 \cos \left(\alpha+\varepsilon+\bar{\alpha}_{e}\left(y_{e_{S}}\right)\right)-2 T \sin \left(\alpha+\varepsilon+\bar{\alpha}_{e}\left(y_{e_{S}}\right)\right) \frac{\partial \bar{\alpha}_{e}\left(y_{e_{S}}\right)}{\partial T} \\
J_{21}=\frac{\partial f_{z_{S}}}{\partial \alpha}=2 T \cos \left(\alpha+\varepsilon+\bar{\alpha}_{e}\left(y_{e_{S}}\right)\right)\left(1+\frac{\partial \bar{\alpha}_{e}\left(y_{e_{S}}\right)}{\partial \alpha}\right)+\left(C_{L_{\alpha}}+\frac{\partial \int C_{L_{\alpha}} \bar{\alpha}_{e} d y_{S}}{\partial \alpha}\right) q_{\infty} S_{r e f}
\end{gathered}
$$




$$
\begin{gathered}
J_{22}=\frac{\partial f_{z_{S}}}{\partial \delta_{e}}=C_{L_{\delta_{e}}} q_{\infty} S_{r e f} \\
J_{23}=\frac{\partial f_{z_{S}}}{\partial T}=2 \sin \left(\alpha+\varepsilon+\bar{\alpha}_{e}\left(y_{e_{S}}\right)\right)+2 \cos \left(\alpha+\varepsilon+\bar{\alpha}_{e}\left(y_{e_{S}}\right)\right) \frac{\partial \bar{\alpha}_{e}\left(y_{e_{S}}\right)}{\partial T}+\frac{\partial \int C_{L_{\alpha}} \bar{\alpha}_{e} d y_{S}}{\partial T} q_{\infty} S_{r e f} \\
J_{31}=\frac{\partial m_{y_{S}}}{\partial \alpha}=C_{m_{\alpha}} q_{\infty} S_{r e f} \bar{c}+\frac{\partial \int C_{m_{\alpha}} \bar{\alpha}_{e} d y_{S}}{\partial \alpha} q_{\infty} S_{r e f} \bar{c} \\
-2 T z_{e} \sin \left(\varepsilon+\bar{\alpha}_{e}\left(y_{e_{S}}\right)\right) \frac{\partial \bar{\alpha}_{e}\left(y_{e_{S}}\right)}{\partial T}+2 T x_{e} \cos \left(\varepsilon+\bar{\alpha}_{e}\left(y_{e_{S}}\right)\right) \frac{\partial \bar{\alpha}_{e}\left(y_{e_{S}}\right)}{\partial T} \\
J_{33}=\frac{\partial m_{y_{S}}}{\partial \delta_{e}}=C_{m_{\delta_{e}}} q_{\infty} S_{r e f} \bar{c} \\
+2 T\left(\cos \left(\varepsilon+\bar{\alpha}_{e}\left(y_{e_{S}}\right)\right) x_{e_{S}}-\sin \left(\varepsilon+\bar{\alpha}_{e}\left(y_{e_{S}}\right)\right) z_{e_{S}}\right) \frac{\partial \bar{\alpha}_{e}\left(y_{e_{S}}\right)}{\partial T}=2\left(\cos \left(\varepsilon+\bar{\alpha}_{e}\left(y_{e_{S}}\right)\right) z_{e_{S}}+\sin \left(\varepsilon+\bar{\alpha}_{e}\left(y_{e_{S}}\right)\right) x_{e_{S}}\right)+\frac{\partial \int C_{m_{\alpha}} \bar{\alpha}_{e}\left(y_{e_{S}}\right) d y}{\partial z_{\infty} S_{r e f} \bar{c}} \\
+2 T\left(\cos \left(\varepsilon+\bar{\alpha}_{e}\left(y_{e_{S}}\right)\right) \frac{\partial z_{e_{S}}}{\partial T}+\sin \left(\varepsilon+\bar{\alpha}_{e}\left(y_{e_{S}}\right)\right) \frac{\partial x_{e_{S}}}{\partial T}\right)
\end{gathered}
$$

The elastic terms in the Jacobian, which require running a static aeroelastic sensitivity study to $\alpha$ and $T$ at each iteration, can be costly to calculate. Therefore, for the scope of this study, these terms are not used in approximation of the Jacobian. The value of $2 K C_{L_{\alpha}}^{2} \alpha$ is also approximated as the value $C_{D_{\alpha}}$, which is rapidly estimated by vortex-lattice code. As a trade-off in using these simplifications, the convergence rate of the trim algorithm is expected to decrease. The Jacobian for the system becomes:

$$
J=\left[\begin{array}{ccc}
-2 T \sin (\alpha+\varepsilon)-C_{D_{\alpha}} q_{\infty} S_{r e f} & -C_{D_{\delta_{e}}} q_{\infty} S_{r e f} & 2 \cos (\alpha+\varepsilon) \\
2 T \cos (\alpha+\varepsilon)+C_{L_{\alpha}} q_{\infty} S_{r e f} & C_{L_{\delta_{e}}} q_{\infty} S_{r e f} & 2 \sin (\alpha+\varepsilon) \\
C_{m_{\alpha}} q_{\infty} S_{r e f} \bar{c} & C_{m_{\delta_{e}}} q_{\infty} S_{r e f} \bar{c} & 2\left(\cos (\varepsilon) z_{e_{S}}+\sin (\varepsilon) x_{e_{S}}\right)
\end{array}\right]
$$

The static aeroelastic framework in Fig. 8 is augmented with an additional step in which the Newton's method trim procedure is added within the structural-aerodynamic loops. The resulting framework is represented in Fig. 10.

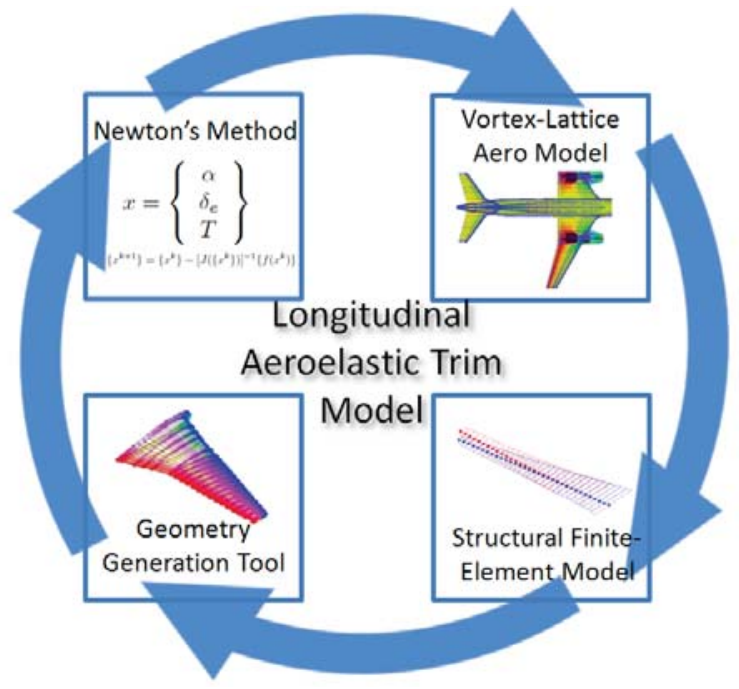

Figure 10. Static Aeroelastic Longitudinal Trim Model

Additional steps and modifications are added to the static aeroelastic approach to generate the aeroelastic trim procedure: 
1. Vortex-lattice modeling is conducted on an input geometry at an estimated trim flight condition to determine the aircraft total aerodynamic quantities and control derivatives $\left(C_{L_{\delta e}}, C_{m_{\delta e}}, C_{D_{\delta e}}\right)$ as well as sectional coefficients $c_{L}(x), c_{m_{a c}}(x), k(x), c_{L_{\alpha}}(x)$, and $x_{a c}(x)$.

2. The structural FEM model uses the sectional aerodynamic load inputs to calculate the wing's structural deflection $\Theta(x)$ and $W(x)$.

3. The geometry generation tool converts $\Theta(x)$ and $W(x)$ into the series of deformations $\alpha_{e}(x), \Delta y_{V}(x)$, and $\Delta z_{V}(x)$, and generates a new aircraft geometry with the deformed wing.

4. Newton's method is used to determine a new trim flight condition and inputs $\alpha, \delta_{e}$, and $T$.

5. Steps 1-4 are repeated until a convergence criteria is met.

The outputs of the static aeroelastic trim code are the flexible aircraft trim inputs $\bar{\alpha}, \bar{\delta}_{e}$ and $\bar{T}$ as well as the aeroelastic deflections $\bar{\Theta}(x), \bar{W}(x), \bar{W}_{x}(x)$, and $\bar{\alpha}_{e}(x)$.

The convergence of the trim code is evaluated based upon the non-dimensionalized values of the forces and moment in the stability axes. Let $\beta_{1}, \beta_{2}$, and $\beta_{3}$ be user defined parameters. The following three equilibrium criteria must be satisfied for a trim solution to be determined:

$$
\begin{aligned}
\frac{f_{x_{S}}}{q_{\infty} S_{\text {ref }}} & \leq\left|\beta_{1}\right| \\
\frac{f_{z_{S}}}{q_{\infty} S_{r e f}} & \leq\left|\beta_{2}\right| \\
\frac{m_{y_{S}}}{q_{\infty} S_{r e f} \bar{c}} & \leq\left|\beta_{3}\right|
\end{aligned}
$$

A convergence criteria is also placed on the aircraft quantity $\left|\Delta\left(C_{L_{0}}+C_{L_{e}}\right)\right|$ such that its value between iterations must also be below a certain threshold. This differs from the static aeroelastic model convergence criteria on $\left|\Delta C_{L}\right|$ because the aircraft angle of attack $\alpha$ is not held constant in trim model. This change in convergence criteria helps to ensure that aeroelastic shape is converged as the trim solution is determined.

The framework in Fig. 10 represents a simplistic aeroelastic trim procedure where Newton's method is added directly into the structural-aerodynamic iterations. Other variations of a trim algorithm could use structural-aerodynamic coupling as an inner loop while Newton's method is wrapped around the static aeroelastic model, thus converging the aeroelastic shape of the wing at each flight condition prior to conducting a Newton's method iteration. These approaches are not investigated in this work.

\section{Simulations and Results}

The following sections summarize the results from simulating the static aeroelastic and aeroelastic longitudinal trim models for different aircraft models. Flexible wing structural and aeroelastic deflections are presented, and the previous used conventions are maintained:

$\Theta(x)$ represents elastic axis twist, positive nose-down.

$W(x)$ represents vertical or flapwise bending, positive upwards.

$W_{x}(x)$ represents vertical or flapwise bending slope, positive corresponding with positive upwards deflection.

$\alpha_{e}(x)$ represents elastic contribution to the angle of attack or twist about the pitch axis, positive nose-up.

Values represented with a bar such as $\bar{\Theta}$ and $\bar{W}$ represent aeroelastic deflections computed through convergence of structural-aerodynamic iterations.

\section{A. Idealized Wing Alone Model, Static Aeroelastic Analysis}

The static aeroelastic code is implemented on the Idealized Wing Alone model as a validation study. The Idealized Wing Alone model is simulated at the $80 \%$ fuel cruise case of the GTM, corresponding to $M=0.8, h=30,000 \mathrm{ft}$, and $W=190,000 \mathrm{lbs}$. Representative mass and stiffness values based on the GTM data are used to generate the beam model for the Idealized Wing Alone, and the empty weight of the wing is simulated to be $W_{w, 0}=17,250 \mathrm{lbs}$. The weight of the engines are simulated on the structural beam model as point loads, but the thrust effect on the structural model are ignored. The aerodynamic model of the Idealized Wing Alone does not include the engine as a simplification. 
Lift curves and drag polars for the Idealized Wing Alone model are generated for different cases and are shown in Fig. 11. The drag polars for the Idealized Wing Alone do not include parasitic drag and only show the vortexlattice computed drag. In the cases where the the wing is considered "rigid", the wing's structural deflections do not have any coupling to the aerodynamic modeling. Thus, for the cases labeled "rigid", the aerodynamics on the model are equivalent to the loads that a rigid planform would experience. The structural deflection results are simply the deflection that would be expected based on the rigid wing loads. These differ from the coupled aeroelastic solutions corresponding to the results labeled as "flexible".
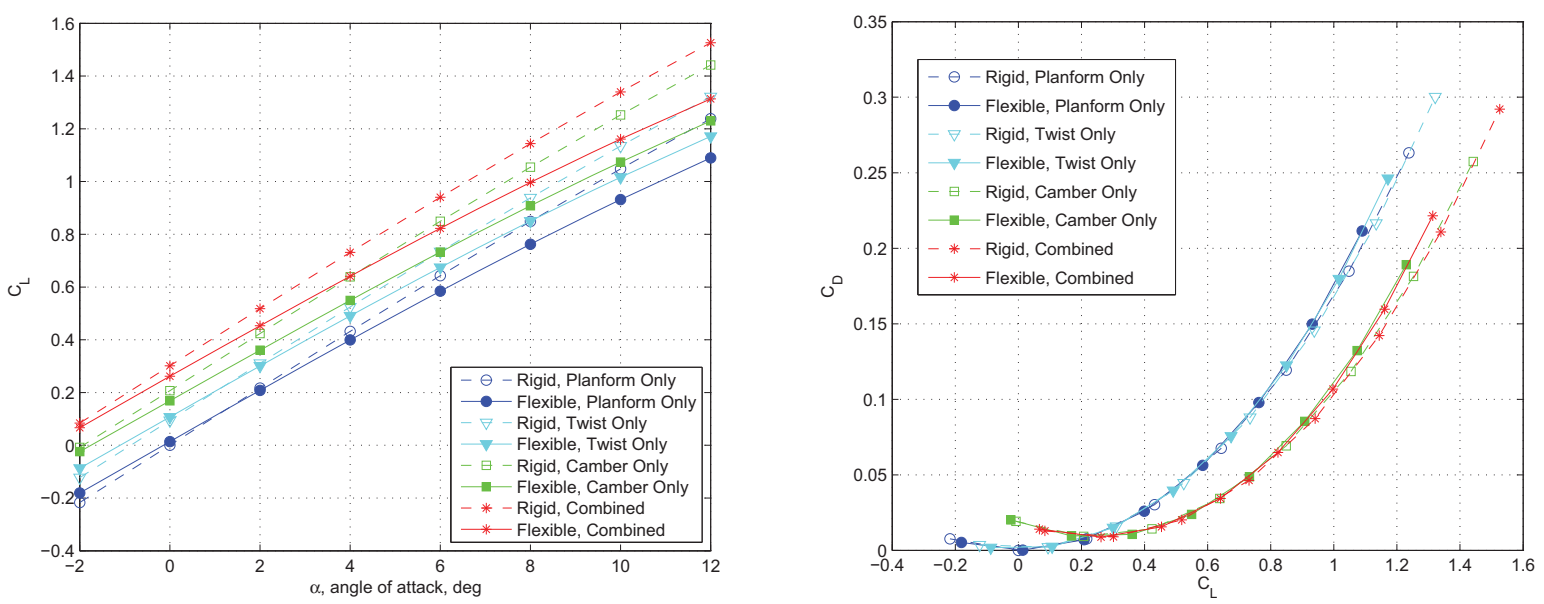

Figure 11. Lift Curves and Drag Polars for Idealized Wing Alone Model

Figure 12 shows the deflection results for the flight condition at $M=0.8$, for the Idealized Wing Alone model. The rigid models' tip deflection $W_{t i p}$ are presented along with the flexible models' aeroelastic tip deflections $\bar{W}_{t i p}$. The tip elastic twist values $\Theta_{t i p}$ and $\bar{\Theta}_{t i p}$ are also presented for the rigid and flexible models.
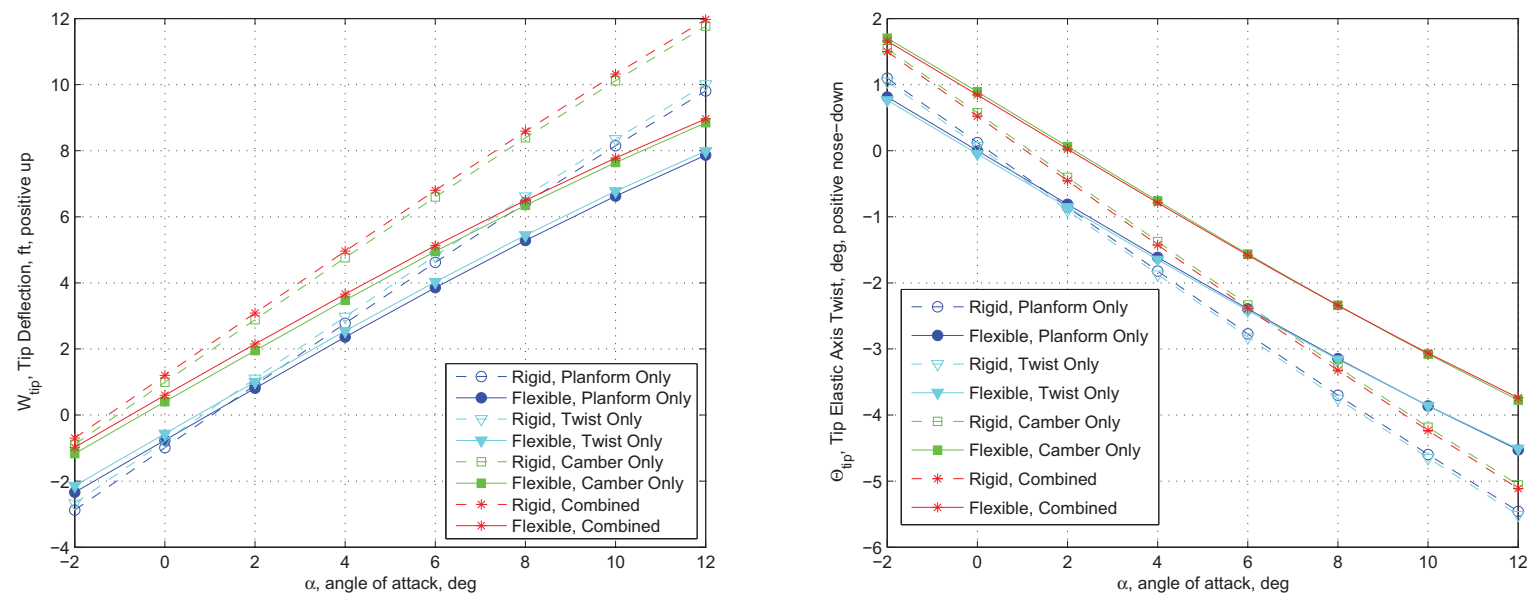

Figure 12. Structural and Aeroelastic Deflections for Idealized Wing Alone Model

Based on the aircraft weight of $W=190,000 \mathrm{lbs}$, a trim $C_{L}$ for a 1-g load is determined for the Idealized Wing Alone to be $C_{L}=0.346$. Because the Idealized Wing Alone is not modeled with a horizontal tail or with engine thrust, a simple angle of attack $\alpha$ trim can be conducted based off the lift curves developed in Fig. 11. The static aeroelastic deflections (or the structural deflections for the rigid wing case) are determined and compared against NASTRAN static aeroelastic results provided by Boeing Research \& Technology. ${ }^{2}$ The results for the rigid wing structural deflection solutions are presented in Table 1 . In addition to the wing tip deflection $W_{\text {tip }}$ and wing tip elastic 
axis twist $\Theta_{t i p}$, the value of the elastic contribution to angle of attack $\alpha_{e, t i p}$ is also provided representing the amount of twist of the wing tip about the pitch axis.

\begin{tabular}{|c|c|c|c|}
\hline Planform Only & Wing Alone Model & NASTRAN & $\begin{array}{c}\text { Absolute } \\
\text { Difference }\end{array}$ \\
\hline \hline$\alpha$, deg & 3.20 & 3.15 & 0.05 \\
\hline$W_{t i p}$, in & 24.34 & 22.42 & 1.92 \\
\hline$\Theta_{t i p}, \operatorname{deg}$ & -1.43 & -1.12 & 0.31 \\
\hline$\alpha_{e, t i p}, \operatorname{deg}$ & -0.25 & -0.36 & 0.11 \\
\hline
\end{tabular}

\begin{tabular}{|c|c|c|c|}
\hline With Twist & Wing Alone Model & NASTRAN & $\begin{array}{c}\text { Absolute } \\
\text { Difference }\end{array}$ \\
\hline \hline$\alpha$, deg & 2.33 & 2.27 & 0.06 \\
\hline$W_{\text {tip }}$, in & 16.97 & 15.12 & 1.85 \\
\hline$\Theta_{t i p}, \operatorname{deg}$ & -1.06 & -0.80 & 0.26 \\
\hline$\alpha_{e, t i p}, \operatorname{deg}$ & -0.07 & -0.16 & 0.09 \\
\hline
\end{tabular}

\begin{tabular}{|c|c|c|c|}
\hline With Camber & Wing Alone Model & NASTRAN & $\begin{array}{c}\text { Absolute } \\
\text { Difference }\end{array}$ \\
\hline \hline$\alpha$, deg & 1.28 & 1.16 & 0.21 \\
\hline$W_{t i p}$, in & 26.50 & 28.28 & 1.78 \\
\hline$\Theta_{t i p}, \operatorname{deg}$ & -0.05 & 0.19 & 0.24 \\
\hline$\alpha_{e, t i p}, \operatorname{deg}$ & -1.64 & -1.94 & 0.30 \\
\hline
\end{tabular}

\begin{tabular}{|c|c|c|c|}
\hline Combined & Wing Alone Model & NASTRAN & $\begin{array}{c}\text { Absolute } \\
\text { Difference }\end{array}$ \\
\hline \hline$\alpha$, deg & 0.41 & 0.29 & 0.12 \\
\hline$W_{t i p}$, in & 19.05 & 20.99 & 1.94 \\
\hline$\Theta_{t i p}, \operatorname{deg}$ & 0.32 & 0.51 & 0.19 \\
\hline$\alpha_{e, t i p}, \operatorname{deg}$ & -1.46 & -1.74 & 0.28 \\
\hline
\end{tabular}

Table 1. Structural Deflection (Rigid Wing) Results for Idealized Wing Alone, $h=30,000 \mathbf{f t}$

The structural deflection results for the Idealized Wing Alone model when only the rigid wing loads are considered are in good agreement between the aeroelastic model and the NASTRAN results. The percent differences in tip deflection between the Idealized Wing Alone model and the NASTRAN results are 8.56\%, 12.2\%, 6.29\%, and 9.24\% respectively for the planform only model, planform and twist, planform and camber, and combined models. Wing tip twist agrees where the absolute differences of $\Theta_{t i p} \leq\left|0.31^{\circ}\right|$ and $\alpha_{e, t i p} \leq\left|0.30^{\circ}\right|$ are observed for elastic axis twist and pitch axis twist, respectively.

The effects of adding twist, camber, and twist and camber on the aeroelastic model with no coupling serve as confirmation of expected aerodynamic behavior. Because the angle of attack $\alpha$ is adjusted to maintain the total load of $W=190,000 \mathrm{lbs}$, the effect of twist, camber, and both combined acts only to redistribute the lift load along the planform of the wing. With just the planform, the angle of attack to maintain $C_{L}=0.346$ is $\alpha=3.20^{\circ}$. With twist enabled, the angle of attack decreases to $\alpha=2.33^{\circ}$. The decrease in angle of attack is expected due to the positive nose-up twist of the jig-shape at the root which increases the net lift on the planform. The wash-out of the wing twist shifts the lift distribution towards the root, and thus, a decrease in the tip deflection $W_{t i p}$ and more nose-down tip twist $\Theta_{t i p}$ are observed. The introduction of camber also significantly affects the angle of attack for $C_{L}=0.346$. It is known from aerodynamics that camber significantly increases the lift on airfoil sections and also introduces moment about the airfoil aerodynamic center. Because the cambered wing is able to generate much more lift than the flat planform, the angle of attack needed to maintain the $1 \mathrm{~g}$ lift load is only $\alpha=1.28^{\circ}$, much less than the planform only. The 
tip twist $\Theta_{t i p}$ also becomes more nose-down. This is expected, as typical cambered aircraft wings have negative $c_{m_{a c}}$ which twists the airfoil sections nose-down.

The structural deflection results without aeroelastic coupling are recomputed for an altitude of $h=35,000 \mathrm{ft}\left(C_{L}=\right.$ 0.437 ) and the results are summarized in Table 2.

\begin{tabular}{|c|c|c|c|}
\hline Planform Only & Wing Alone Model & NASTRAN & $\begin{array}{c}\text { Absolute } \\
\text { Difference }\end{array}$ \\
\hline \hline$\alpha$, deg & 4.04 & 3.98 & 0.06 \\
\hline$W_{\text {tip }}$, in & 24.34 & 22.42 & 1.92 \\
\hline$\Theta_{t i p}, \operatorname{deg}$ & -1.43 & -1.12 & 0.31 \\
\hline$\alpha_{e, t i p}, \operatorname{deg}$ & -0.25 & -0.36 & 0.11 \\
\hline
\end{tabular}

\begin{tabular}{|c|c|c|c|}
\hline With Twist & Wing Alone Model & NASTRAN & $\begin{array}{c}\text { Absolute } \\
\text { Difference }\end{array}$ \\
\hline \hline$\alpha$, deg & 3.17 & 3.09 & 0.08 \\
\hline$W_{\text {tip }}$, in & 18.53 & 16.63 & 1.90 \\
\hline$\Theta_{t i p}, \operatorname{deg}$ & -1.14 & -0.86 & 0.28 \\
\hline$\alpha_{e, t i p}, \operatorname{deg}$ & -0.10 & -0.20 & 0.10 \\
\hline
\end{tabular}

\begin{tabular}{|c|c|c|c|}
\hline With Camber & Wing Alone Model & NASTRAN & $\begin{array}{c}\text { Absolute } \\
\text { Difference }\end{array}$ \\
\hline \hline$\alpha$, deg & 2.12 & 1.99 & 0.13 \\
\hline$W_{t i p}$, in & 26.03 & 27.07 & 1.04 \\
\hline$\Theta_{t i p}, \operatorname{deg}$ & -0.34 & -0.08 & 0.26 \\
\hline$\alpha_{e, t i p}, \operatorname{deg}$ & -1.35 & -1.61 & 0.26 \\
\hline
\end{tabular}

\begin{tabular}{|c|c|c|c|}
\hline Combined & Wing Alone Model & NASTRAN & $\begin{array}{c}\text { Absolute } \\
\text { Difference }\end{array}$ \\
\hline \hline$\alpha$, deg & 1.25 & 1.10 & 0.15 \\
\hline$W_{t i p}$, in & 20.18 & 21.28 & 1.10 \\
\hline$\Theta_{t i p}, \operatorname{deg}$ & -0.04 & 0.18 & 0.22 \\
\hline$\alpha_{e, t i p}, \operatorname{deg}$ & -1.21 & -1.46 & 0.25 \\
\hline
\end{tabular}

Table 2. Structural Deflection (Rigid Wing) Results for Idealized Wing Alone, $h=35,000 \mathbf{f t}$

Good agreement still exists at the increased altitude of $h=35,000 \mathrm{ft}$ between the developed aeroelastic model and NASTRAN results for the Idealized Wing Alone model when only the rigid wing loads are considered. Percent differences of $8.56 \%, 11.4 \%, 3.84 \%$, and $5.26 \%$ respectively for the planform only model, planform and twist, planform and camber, and combined models are observed between the wing tip deflection $W_{\text {tip }}$ results with the developed aeroelastic model and NASTRAN. Absolute differences of $\Theta_{t i p} \leq\left|0.31^{\circ}\right|$ and $\alpha_{e, t i p} \leq\left|0.26^{\circ}\right|$ are observed for wing tip elastic axis twist and pitch axis twist, respectively.

The aerodynamic trends observed for the Idealized Wing Alone model with the rigid wing at $h=30,000 \mathrm{ft}$ are consistent with the results at $h=35,000 \mathrm{ft}$. The addition of twist, camber, and twist and camber to the planform increases the lift generated by the model and subsequently reduces the angle of attack $\alpha$ needed to maintain a load of $C_{L}=0.346$. 
The results when coupling is enabled and the framework is true to Fig. 8 is presented in Table 3 for $C_{L}=0.346$ at $h=30,000 \mathrm{ft}$.

\begin{tabular}{|c|c|c|c|}
\hline Planform Only & Wing Alone Model & NASTRAN & $\begin{array}{c}\text { Absolute } \\
\text { Difference }\end{array}$ \\
\hline \hline$\alpha$, deg & 3.44 & 3.41 & 0.03 \\
\hline $\bar{W}_{t i p}$, in & 23.13 & 21.28 & 1.85 \\
\hline $\bar{\Theta}_{t i p}, \operatorname{deg}$ & -1.39 & -1.08 & 0.31 \\
\hline $\bar{\alpha}_{e, t i p}, \operatorname{deg}$ & -0.21 & -0.33 & 0.12 \\
\hline
\end{tabular}

\begin{tabular}{|c|c|c|c|}
\hline With Twist & Wing Alone Model & NASTRAN & $\begin{array}{c}\text { Absolute } \\
\text { Difference }\end{array}$ \\
\hline \hline$\alpha, \operatorname{deg}$ & 2.47 & 2.42 & 0.05 \\
\hline $\bar{W}_{t i p}$, in & 16.43 & 14.62 & 1.81 \\
\hline $\bar{\Theta}_{t i p}, \operatorname{deg}$ & -1.04 & -0.78 & 0.26 \\
\hline $\bar{\alpha}_{e, t i p}, \operatorname{deg}$ & -0.05 & -0.15 & 0.10 \\
\hline
\end{tabular}

\begin{tabular}{|c|c|c|c|}
\hline With Camber & Wing Alone Model & NASTRAN & $\begin{array}{c}\text { Absolute } \\
\text { Difference }\end{array}$ \\
\hline \hline$\alpha, \operatorname{deg}$ & 1.84 & 1.88 & 0.04 \\
\hline $\bar{W}_{t i p}$, in & 22.07 & 23.43 & 1.36 \\
\hline $\bar{\Theta}_{t i p}, \operatorname{deg}$ & 0.12 & 0.38 & 0.26 \\
\hline $\bar{\alpha}_{e, t i p}, \operatorname{deg}$ & -1.49 & -1.79 & 0.30 \\
\hline
\end{tabular}

\begin{tabular}{|c|c|c|c|}
\hline Combined & Wing Alone Model & NASTRAN & $\begin{array}{c}\text { Absolute } \\
\text { Difference }\end{array}$ \\
\hline \hline$\alpha$, deg & 0.87 & 0.90 & 0.03 \\
\hline $\bar{W}_{t i p}$, in & 15.34 & 16.78 & 1.44 \\
\hline $\bar{\Theta}_{t i p}, \operatorname{deg}$ & 0.49 & 0.68 & 0.19 \\
\hline $\bar{\alpha}_{e, t i p}, \operatorname{deg}$ & -1.35 & -1.62 & 0.27 \\
\hline
\end{tabular}

Table 3. Aeroelastic Deflection (Flexible Wing) Results for Idealized Wing Alone, $h=30,000 \mathrm{ft}$

The good agreement between the aeroelastic Idealized Wing Alone model and the NASTRAN aeroelastic model results remains when structural-aerodynamic coupling is enabled. Percent differences of $8.69 \%, 12.3 \%, 5.80 \%$, and $8.58 \%$ between the wing tip deflection $W_{t i p}$ results of the developed aeroelastic model and the NASTRAN model are observed for the planform only, planform and twist, planform and camber, and combined models, respectively. For wing tip twist, absolute differences of $\Theta_{t i p} \leq\left|0.31^{\circ}\right|$ and $\alpha_{e, t i p} \leq\left|0.30^{\circ}\right|$ are observed for wing tip elastic axis twist and pitch axis twist, respectively.

The results in Table 3 can also be compared against the results in Table 1 to illustrate the effect of adding in aeroelastic coupling on the model. It is observed that the angle of attacks $\alpha$ for the flexible models at $C_{L}=0.346$ are higher than that of the rigid models. This is due to the fact that the aeroelastic deflection on the wings tends to cause flexible wings to twist downwards with a negative $\alpha_{e}$. This reduces the amount of lift generated by the wing at a particular angle of attack $\alpha$. Thus, in order to maintain the same load as a rigid model, a flexible model needs to operate at a higher angle of attack $\alpha$. For example, in the combined Idealized Wing Alone model where twist and camber are both considered, a higher angle of attack $\alpha=0.86^{\circ}$ is required for the flexible model while a lower angle of attack $\alpha=0.41^{\circ}$ is adequate for the rigid model for $C_{L}=0.346$. This phenomenon is also illustrated in Fig. 11, where the flexible wing lift curves are shifted below that of the rigid wing lift curves. The exception is for the uncambered models at low angles of attacks, where the elastic contribution to the aeroelastic angle of attack $\alpha_{e}$ can actually be positive, twisting the wing nose-up. This is due to the fact that the flapwise bending slope $W_{x}$, which 
generally contributes to the nose-down elastic twist of the wing, is very low at these flight conditions.

The results of the static aeroelastic model for the increased altitude $h=35,000 \mathrm{ft}$ or $C_{L}=0.437$ flight condition are also compared against NASTRAN results.

\begin{tabular}{|c|c|c|c|}
\hline Planform Only & Wing Alone Model & NASTRAN & $\begin{array}{c}\text { Absolute } \\
\text { Difference }\end{array}$ \\
\hline \hline$\alpha$, deg & 4.29 & 4.23 & 0.06 \\
\hline $\bar{W}_{t i p}$, in & 23.36 & 21.50 & 1.86 \\
\hline $\bar{\Theta}_{t i p}, \operatorname{deg}$ & -1.40 & -1.09 & 0.31 \\
\hline $\bar{\alpha}_{e, t i p}, \operatorname{deg}$ & -0.21 & -0.33 & 0.21 \\
\hline
\end{tabular}

\begin{tabular}{|c|c|c|c|}
\hline With Twist & Wing Alone Model & NASTRAN & $\begin{array}{c}\text { Absolute } \\
\text { Difference }\end{array}$ \\
\hline \hline$\alpha, \operatorname{deg}$ & 3.34 & 3.27 & 0.07 \\
\hline $\bar{W}_{\text {tip }}$, in & 17.98 & 16.13 & 1.85 \\
\hline $\bar{\Theta}_{\text {tip }}, \operatorname{deg}$ & -1.12 & -0.85 & 0.27 \\
\hline $\bar{\alpha}_{e, t i p}, \operatorname{deg}$ & -0.09 & -0.19 & 0.10 \\
\hline
\end{tabular}

\begin{tabular}{|c|c|c|c|}
\hline With Camber & Wing Alone Model & NASTRAN & $\begin{array}{c}\text { Absolute } \\
\text { Difference }\end{array}$ \\
\hline \hline$\alpha, \operatorname{deg}$ & 2.64 & 2.62 & 0.02 \\
\hline $\bar{W}_{\text {tip }}$, in & 22.90 & 23.77 & 0.87 \\
\hline $\bar{\Theta}_{\text {tip }}, \operatorname{deg}$ & -0.22 & 0.04 & 0.26 \\
\hline $\bar{\alpha}_{e, t i p}, \operatorname{deg}$ & -1.24 & -1.51 & 0.27 \\
\hline
\end{tabular}

\begin{tabular}{|c|c|c|c|}
\hline Combined & Wing Alone Model & NASTRAN & $\begin{array}{c}\text { Absolute } \\
\text { Difference }\end{array}$ \\
\hline \hline$\alpha$, deg & 1.67 & 1.66 & 0.01 \\
\hline $\bar{W}_{t i p}$, in & 17.57 & 18.40 & 0.83 \\
\hline $\bar{\Theta}_{t i p}, \operatorname{deg}$ & 0.07 & 0.29 & 0.22 \\
\hline $\bar{\alpha}_{e, t i p}, \operatorname{deg}$ & -1.13 & -1.37 & 0.24 \\
\hline
\end{tabular}

Table 4. Aeroelastic Deflection (Flexible Wing) Results for Idealized Wing Alone, $h=35,000 \mathrm{ft}$

Good agreement continues to exist between the results of the developed aeroelastic model and that of the NASTRAN generated results. The observed relative percent differences between aeroelastic wing tip deflections are $8.65 \%$, $11.5 \%, 3.66 \%$, and $4.51 \%$ respectively for the planform only model, planform and twist, planform and camber. Absolute differences in aeroelastic wing tip twist about the elastic axis are observed to be $\left|\Delta \bar{\Theta}_{t i p}\right| \leq 0.31^{\circ}$, and absolute differences in the elastic contribution to aeroelastic angle of attack are observed to be $\left|\Delta \bar{\alpha}_{e, t i p}\right| \leq 0.26^{\circ}$.

Based on the results in Tables 1-4, it is observed that the elastic axis twist of the developed aeroelastic model consistently experiences more nose-up twist than that of the NASTRAN results. Nonetheless, the good agreement for all the test cases produces confidence in the aeroelastic framework developed and its predictive capability for flexible wing aircraft. 


\section{B. ESAC Model, Static Aeroelastic and Longitudinal Trim Analysis}

With preliminary validation of the aeroelastic modeling completed, the static aeroelastic framework is extended to that of the ESAC. The ESAC model is representative of a full aircraft configuration with engine nacelles, pylons, tail surfaces, and fuselage present in the modeling.

Two independent and separate cruise conditions are simulated with the ESAC, representing different possible design cruise condition candidates. The weight model is also adjusted based on the different candidate cruise conditions.

- A first cruise condition is simulated at $M=0.8, h=30,000 \mathrm{ft}$, where the $80 \%$ fuel case of the aircraft is $W=190,000 \mathrm{lbs}$ and the empty wing mass is $W_{w, 0}=17,250 \mathrm{lbs}$. The design lift coefficient in this case is $C_{L}=0.346$.

- A second cruise condition is simulated at $M=0.8, h=36,000 \mathrm{ft}$, where the $80 \%$ fuel case of the aircraft is $W=210,000 \mathrm{lbs}$ and the empty wing mass is $W_{w, 0}=13,000 \mathrm{lbs}$. The design lift coefficient for this case is $C_{L}=0.510$.

For the cruise flight conditions, the parasitic drag coefficient is built up using a critical Reynold's number of $\operatorname{Re}_{c}=$ 600,000 . The results are presented in Table 5.

\begin{tabular}{|c|c|c|}
\hline \multicolumn{3}{|c|}{$h=30,000 \mathrm{ft}$} \\
\hline Component & $C_{f}$ & $C_{D_{0}}$ \\
\hline \hline Wings & 0.0037 & 0.0054 \\
\hline Fuselage & 0.0042 & 0.0047 \\
\hline Horizontal Tail & 0.0010 & 0.0015 \\
\hline Vertical Tail & 0.0008 & 0.0011 \\
\hline Engine Nacelles+Pylons & 0.0004 & 0.0010 \\
\hline Total & 0.0102 & 0.0137 \\
\hline
\end{tabular}

\begin{tabular}{|c|c|c|}
\hline \multicolumn{3}{|c|}{$h=36,000 \mathrm{ft}$} \\
\hline Component & $C_{f}$ & $C_{D_{0}}$ \\
\hline \hline Wings & 0.0038 & 0.0056 \\
\hline Fuselage & 0.0044 & 0.0049 \\
\hline Horizontal Tail & 0.0011 & 0.0015 \\
\hline Vertical Tail & 0.0008 & 0.0012 \\
\hline Engine Nacelles+Pylons & 0.0005 & 0.0011 \\
\hline Total & 0.0106 & 0.0142 \\
\hline
\end{tabular}

Table 5. Parasite Drag Build-Up At Cruise Conditions

Initially neglecting thrust and its effect on the aeroelastic deformation, the lift curve and drag polars for the ESAC are generated and shown in Fig. 13. Note that now the drag polar includes the conceptually estimated parasitic drag coefficient.
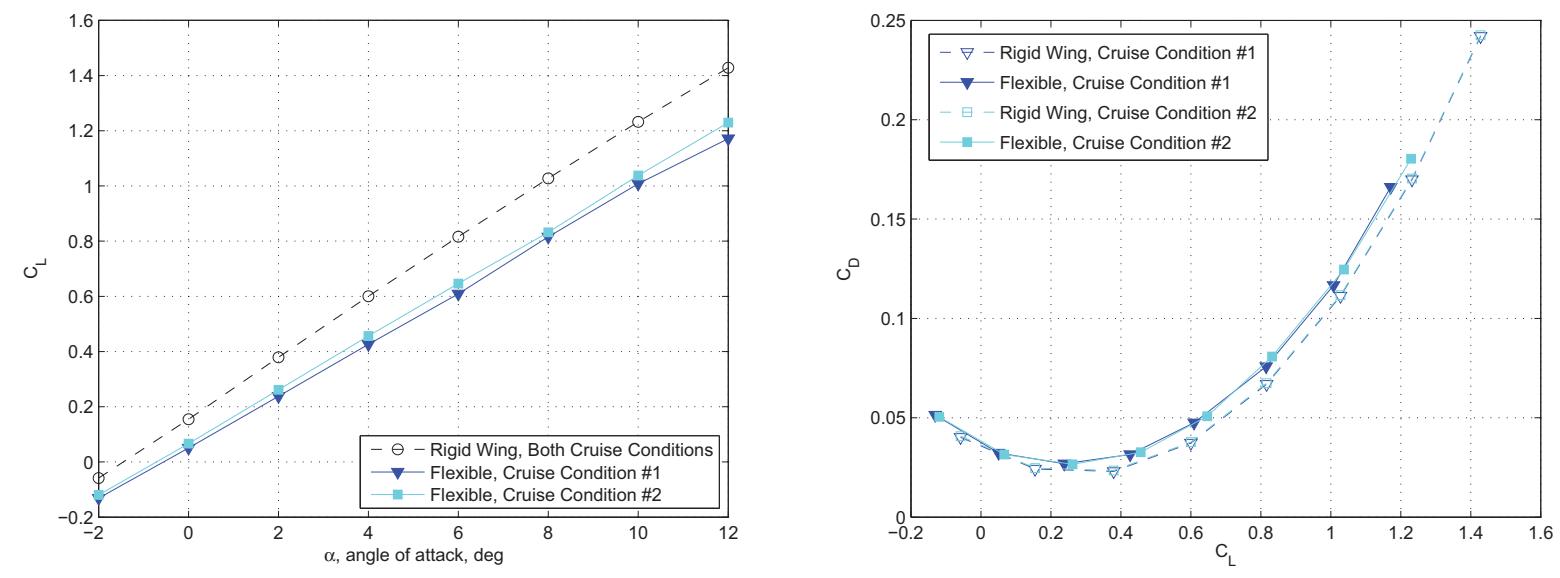

Figure 13. Lift Curves and Drag Polars for ESAC Model, No Thrust or Control Deflection

The tip deflection, $W_{t i p}$ for the rigid model and $\bar{W}_{t i p}$ for the flexible model, and tip elastic axis twist, $\Theta_{t i p}$ for the rigid model and $\bar{\Theta}_{t i p}$ for the flexible model, at the two different flight conditions are shown in Fig. 14. 

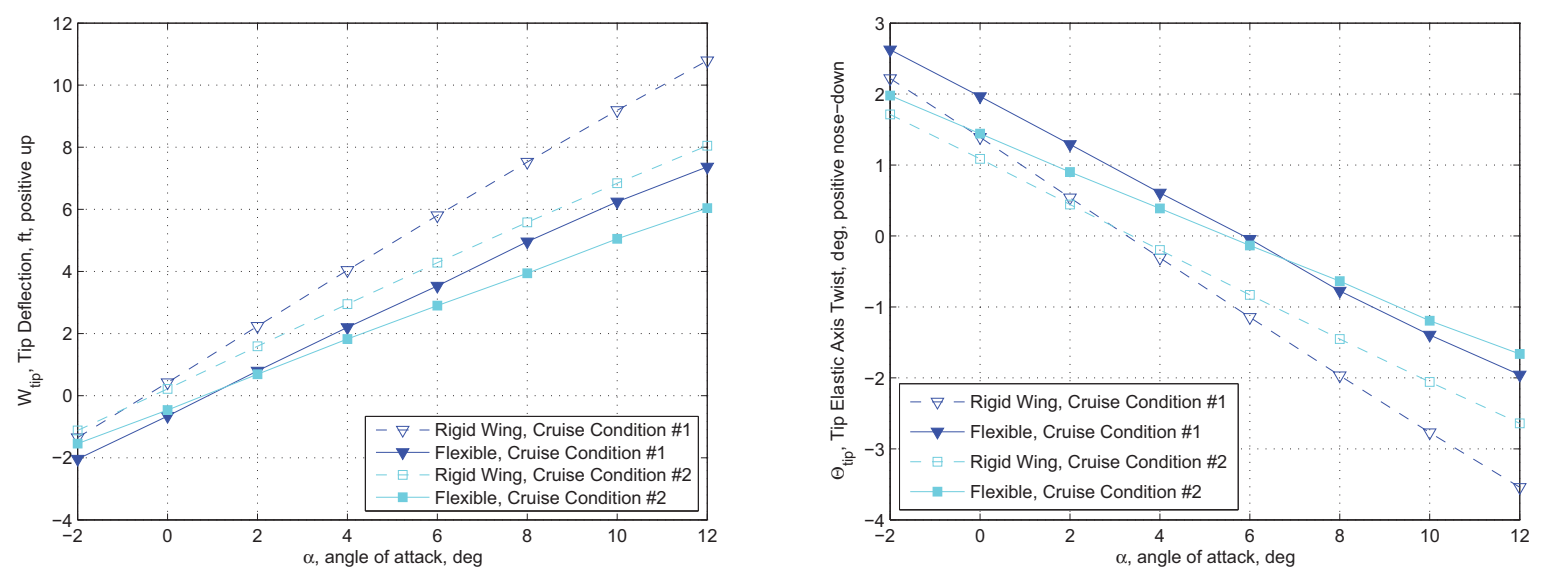

Figure 14. Structural and Aeroelastic Deflections for ESAC Model, No Thrust or Control Deflection

The plots in Fig. 13 and 14 are consistent with expected behavior. The lift curve of the flexible wing models shifts below the rigid wing models due to the aeroelastic deformation's effect on twisting the flexible wing nose down, effectively decreasing the local angle of attack along the wing span. The effect of coupling structural deflection with aerodynamic vortex-lattice also resulted in effective stiffening in bending and softening in torsion. This is observed by the decrease in flapwise deflection in comparing the flexible wing models to the rigid wing models and the increase in nose-down twist.

The longitudinal trim framework is then applied to the ESAC model configuration, and control deflections of the elevator are enabled as well as thrust from the two engines. Deflection of the elevator surface is assumed not to affect the aerodynamics over the wing, but the thrust effect on the wing is considered. The trim algorithm framework can be readily applied to both the rigid and flexible wing models, and the rigid wing model is analyzed first for comparison. The convergence criteria for the trim algorithm is set such that $\beta_{1}=\beta_{2}=0.001$ in Eqs. 109 and 110 and $\beta_{3}=0.00005$ in Eq. 111.

The static trim results for the two cruise conditions when the ESAC's wings are considered rigid are presented in Table 6. Once again, the deflection results presented for the rigid wing solution represent structural deflections assuming that the deformation of the wings does not affect the aerodynamics.

\begin{tabular}{|c|c|}
\hline Cruise Condition \#1 & Design $C_{L}=0.346$ \\
\hline$\alpha, \operatorname{deg}$ & 2.108 \\
\hline$\delta_{e}, \operatorname{deg}$ & -5.075 \\
\hline$T, \mathrm{lb}_{\mathrm{f}}$ (single engine) & 5821 \\
\hline $\mathrm{L} / \mathrm{D}$ & 14.8 \\
\hline$W_{\text {tip }}$, in & 28.082 \\
\hline$\Theta_{t i p}, \operatorname{deg}$ & 0.459 \\
\hline$\alpha_{e, t i p}, \operatorname{deg}$ & -2.209 \\
\hline
\end{tabular}

\begin{tabular}{|c|c|}
\hline Cruise Condition \#2 & Design $C_{L}=0.510$ \\
\hline$\alpha, \mathrm{deg}$ & 3.640 \\
\hline$\delta_{e}, \mathrm{deg}$ & -6.254 \\
\hline$T, \mathrm{lb}_{\mathrm{f}}($ single engine) & 6249 \\
\hline $\mathrm{L} / \mathrm{D}$ & 14.8 \\
\hline$W_{t i p}$, in & 30.810 \\
\hline$\Theta_{\text {tip }}, \mathrm{deg}$ & -0.125 \\
\hline$\alpha_{e, t i p}, \mathrm{deg}$ & 1.841 \\
\hline
\end{tabular}

Table 6. Static Trim Results For Rigid Wing ESAC

The rigid wing ESAC model's trim solution is very easily determined by the trim code, and a total of three iterations of Newton's method were conducted from a user specified estimated initial point of $\alpha=2.0^{\circ}$ for the first cruise condition of design $C_{L}=0.346$ and the initial point of $\alpha=3.0^{\circ}$ for the second cruise condition. The pilot input trim states at each of these iterations are plotted and shown in Fig. 15. 

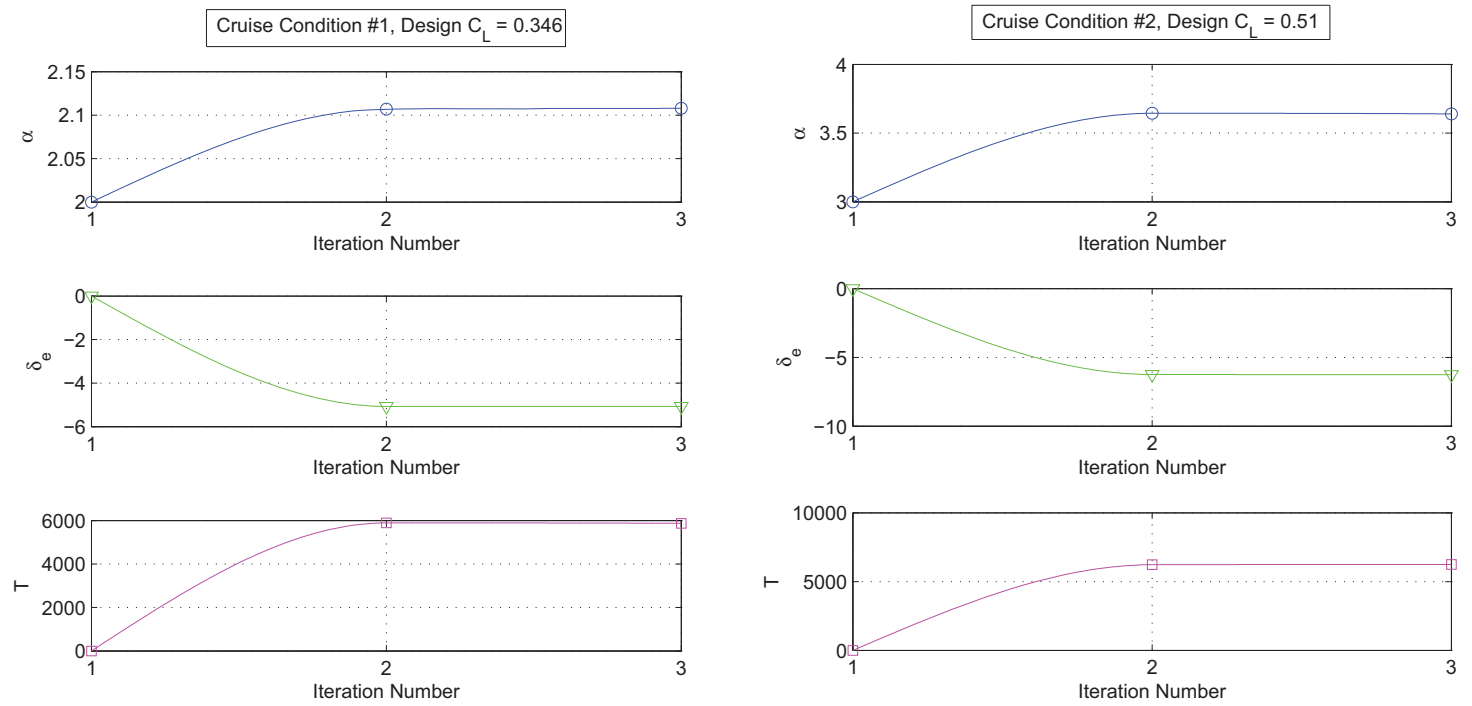

Figure 15. Static Trim Iteration Data for Rigid Wing ESAC

The longitudinal trim code is then run on the ESAC with fully flexible wings. The trim solution is determined, and the results are summarized in Table 7.

\begin{tabular}{|c|c|}
\hline Cruise Condition \#1 & Design $C_{L}=0.346$ \\
\hline$\alpha, \operatorname{deg}$ & 3.747 \\
\hline$\delta_{e}$, deg & -6.798 \\
\hline$T, \mathrm{lb}_{\mathrm{f}}($ single engine) & 7054 \\
\hline L/D & 11.4 \\
\hline $\bar{W}_{t i p}$, in & 24.645 \\
\hline $\bar{\Theta}_{t i p}, \operatorname{deg}$ & 0.643 \\
\hline $\bar{\alpha}_{e, t i p}, \operatorname{deg}$ & -2.159 \\
\hline
\end{tabular}

\begin{tabular}{|c|c|}
\hline Cruise Condition \#2 & Design $C_{L}=0.510$ \\
\hline$\alpha, \operatorname{deg}$ & 5.160 \\
\hline$\delta_{e}, \operatorname{deg}$ & -7.880 \\
\hline$T, \mathrm{lb}_{\mathrm{f}}$ (single engine) & 7285 \\
\hline $\mathrm{L} / \mathrm{D}$ & 11.9 \\
\hline $\bar{W}_{t i p}$, in & 28.469 \\
\hline $\bar{\Theta}_{t i p}, \operatorname{deg}$ & 0.014 \\
\hline $\bar{\alpha}_{e, t i p}, \operatorname{deg}$ & -1.817 \\
\hline
\end{tabular}

Table 7. Static Trim Results For Flexible Wing ESAC

Due to the flexibility of the wing, the trim code takes longer to converge, with 20 iterations for the design $C_{L}=$ 0.346, and 14 iterations for the design $C_{L}=0.510$. The pilot input trim states at each of these iterations are plotted in Fig. 16. As expected, the trim angle of attacks $\alpha$ for the flexible models are higher than that of the rigid models due to the effect of the aeroelastic bending and torsion on the aeroelastic angle of attack.

Because the flexible trim case also involves determining the coupled aeroelastic wing deformation, plots of the evolution of the tip deformation are shown in Fig. 17, where $W_{t i p}$ is plotted in feet and $\Theta_{t i p}$ is plotted in degrees. It is observed that the aeroelastic deformation of the model converges alongside the solution of the static trim inputs. 

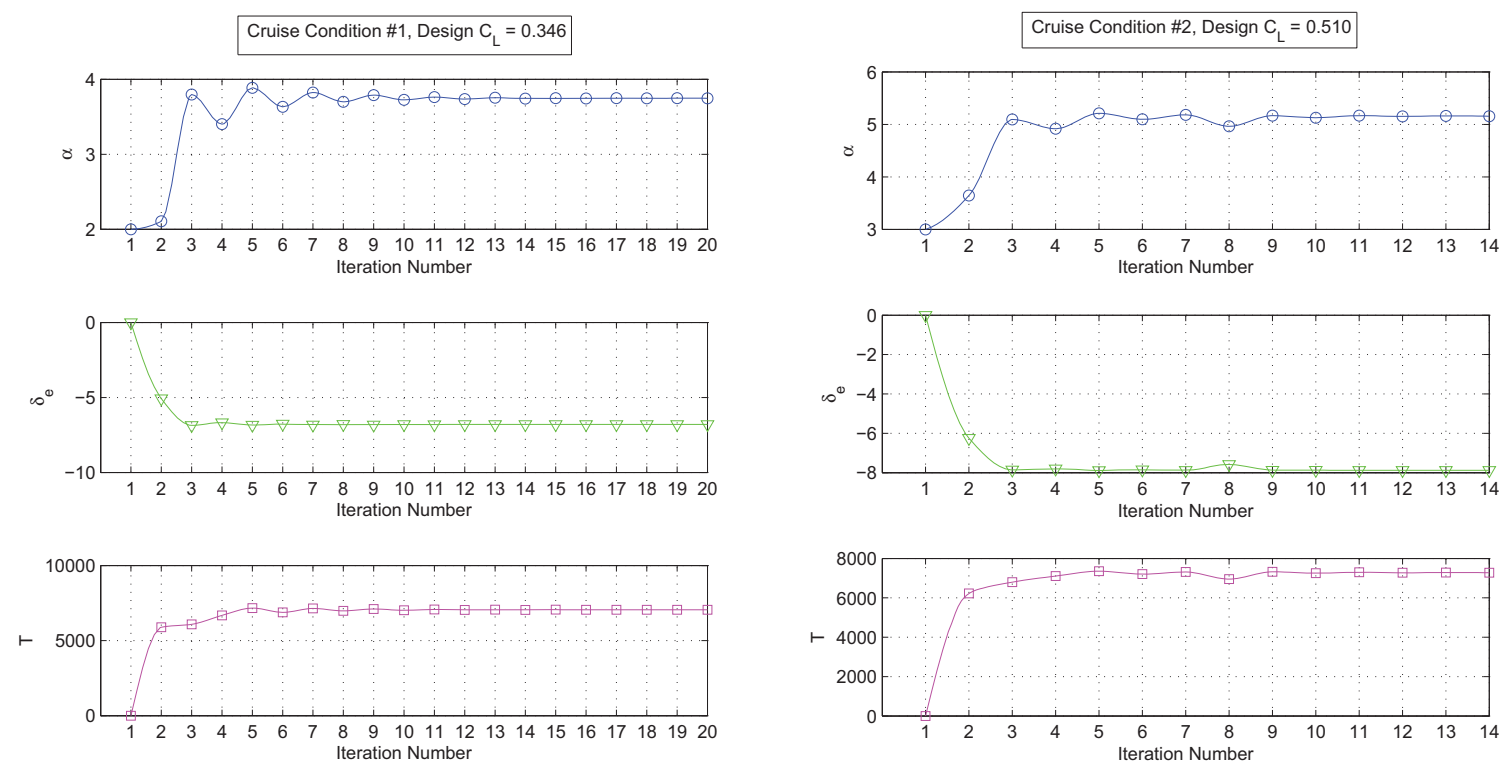

Figure 16. Static Trim Iteration Data for Flexible Wing ESAC
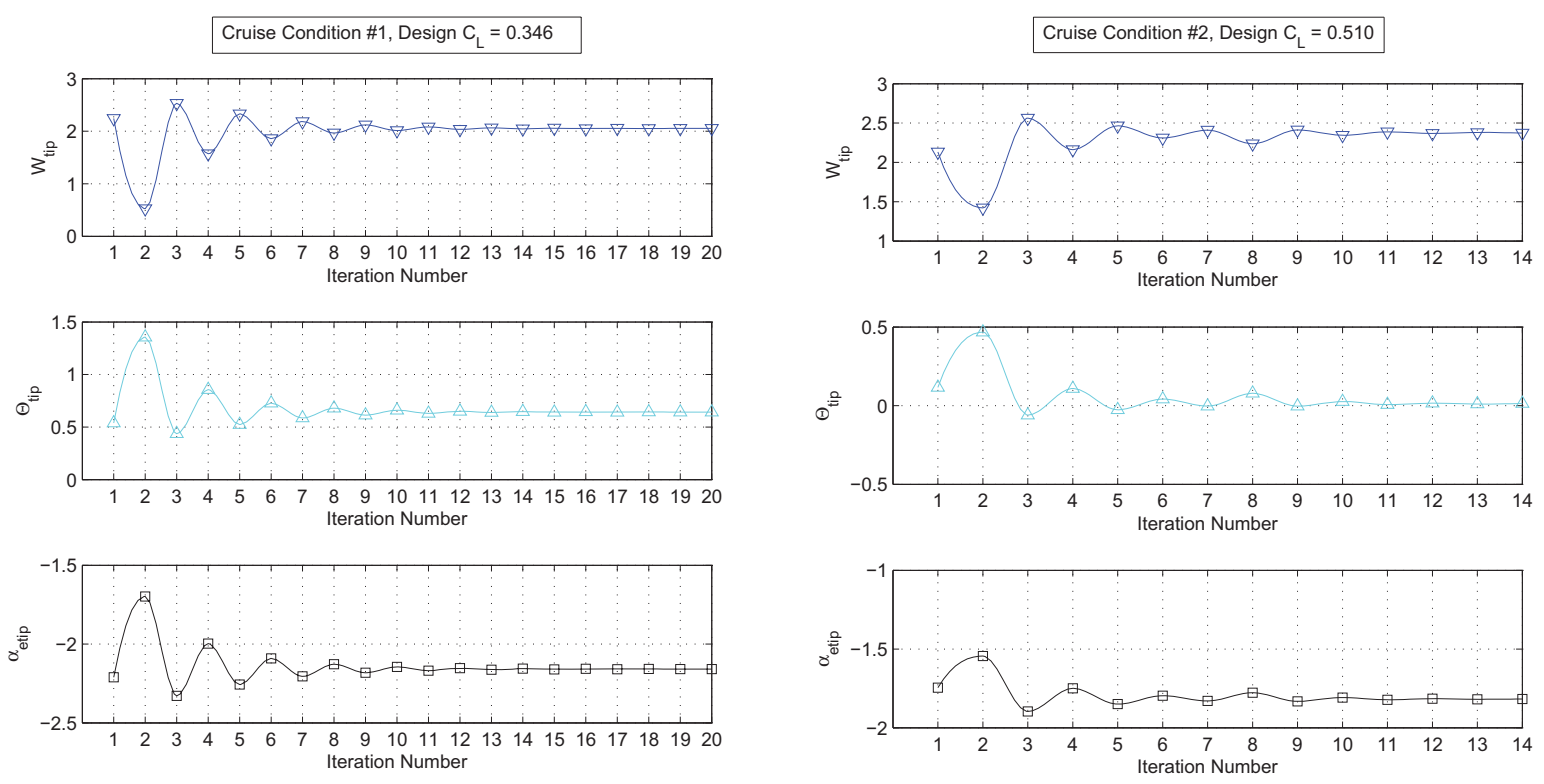

Figure 17. Static Trim Deflection Iteration Data for Flexible Wing ESAC

\section{Conclusions}

This study presents the development of a static aeroelastic model and a static three degree-of-freedom longitudinal aeroelastic trim model capable of analyzing flexible wing aircraft. The aeroelastic model is developed by coupling equivalent beam wing structural models in the loop with vortex-lattice aerodynamic modeling. The static aeroelastic code is capable of generating flexible wing aircraft configurations and characterizing aeroelastic deformations. Utilizing converged aeroelastic geometry, flexible aircraft lift curves and drag polars can be generated exploring the effect of flexibility enabled models. The coupled finite-element vortex-lattice aeroelastic model is validated against NASTRAN aeroelasticity results for a simplified Idealized Wing Alone model for various test cases, including systematically ana- 
lyzing the model planform only, with only added twist, with only added camber, and both added. The static aeroelastic model is extended to develop an aeroelastic three degree-of-freedom longitudinal trim model for a full aircraft configuration with horizontal tail, elevator, and engines. The trim algorithm uses Newton's method which is inserted in the static aeroelastic approach to iterate pilot trim inputs and to solve the flight condition until convergence is achieved. This framework is readily applied for the flexible wing models investigated in this study, but could be improved in the future for highly flexible wings. Future study of an aeroelastic trim model where Newton's method is wrapped around an inner loop static aeroelastic model can be investigated. In addition, different approaches for estimating the Jacobian and variations to Newton's method can possibly increase the convergence rate of the longitudinal trim model.

\section{Acknowledgments}

The authors wish to acknowledge Mr. John Dykman of Boeing Research \& Technology in Seal Beach, CA for providing insight and the NASTRAN results used in development of the models. The authors would also like to thank the NASA Aeronautics Research Mission Directorate (ARMD) Fixed Wing Project under the Fundamental Aeronautics Program for providing the funding to support this work.

\section{References}

\footnotetext{
${ }^{1}$ Nguyen, N., "NASA Innovation Fund 2010 Project: Elastically Shaped Future Air Vehicle Concept," NASA Internal Report Submitted to NASA Innovative Partnerships Program Office, October 8, 2010.

${ }^{2}$ Boeing Report No. 2010X0015, "Development of Variable Camber Continuous Trailing Edge Flap System," submitted to NASA ARMD Fixed Wing Project, October 4, 2012.

${ }^{3}$ Urnes, Sr., J., Nguyen, N., Ippolito, C., Totah, J., Trinh, K., Ting, E., “A Mission-Adaptive Variable Camber Flap Control System to Optimize High Lift and Cruise Lift-to-Drag Ratios of Future N+3 Transport Aircraft," 51st AIAA Aerospace Sciences Meeting, AIAA-2013-0214, January 2013.

${ }^{4}$ Nguyen, N., Trinh, K., Frost S., and Reynolds, K., "Coupled Aeroelastic Vortex Lattice Modeling of Flexible Aircraft," AIAA Applied Aerodynamics Conference, AIAA-2011-3021, June 2011.

${ }^{5}$ Nguyen, N., Trinh, K., Nguyen, D., Tuzcu, I., "Nonlinear Aeroelasticty of Flexible Wing Structure Coupled with Aircraft Flight Dynamics," AIAA Structures, Structural Dynamics, and Materials Conference, AIAA-2012-1792, April 2012.

${ }^{6}$ Meirovitch, L., Fundamentals of Vibrations, McGraw-Hill Higher Education, 2001.

${ }^{7}$ Hodges, D.H. and Pierce, G.A., Introduction to Structural Dynamics and Aeroelasticity, Cambridge University Press, 2002.

${ }^{8}$ Hughes, T., The Finite Element Method: Linear Static and Dynamic Finite Element Analysis, Prentice Hall, Inc., 1987.

${ }^{9}$ Craig, Jr., R.R. and Kurdila, A.J., Fundamentals of Structural Dynamics, Second Edition, John Wiley \& Sons, Inc., 2006.

${ }^{10}$ Jordan, T. L., Langford, W. M., Belcastro, C. M., Foster, J. M., Shah, G. H., Howland, G., and Kidd, R., "Development of a Dynamically Scaled Generic Transport Model Testbest for Flight Research Experiments," AUVSI Unmanned Unlimited, Arlington, VA, 2004.

${ }^{11}$ Nguyen, N., Nelson, A., and Pulliam, T., "Damage Adaptive Control System Research Report," Internal NASA Report, April 2006.

${ }^{12}$ Nguyen, N., "Integrated Flight Dynamics Modeling of Flexible Aircraft with Inertial Force-Propulsion - Aeroelastic Couplings," 46th AIAA Aerospace Sciences Meeting and Exhibit, AIAA-2008-194, January 2008.

${ }^{13}$ Houbolt, J. C and Brooks, G. W., "Differential Equations of Motion for Combined Flapwise Bending, Chordwise Bending, and Torsion of Twisted Nonuniform Rotor Blades,” NACA Technical Note 3905, February 1957.

${ }^{14}$ Miranda, L.R., Elliot, R.D., and Baker, W.M., "A Generalized Vortex Lattice Method for Subsonic and Supersonic Flow Applications," NASA CR-2865, 1977.

${ }^{15}$ Aftosmis, M.J., Berger, M.J., and Melton, J.E., "Robust and Efficient CartesianMesh Generation for Component- Based Geometry," AIAA Journal, Vol. 36, No. 6, 1998, pp. 953-960.

${ }^{16}$ Raymer, D. P., Aircraft Design: A Conceptual Approach, American Institue of Aeronautics and Astronautics, 1989.

${ }^{17}$ Pozrikidis, C., Numerical Computation in Science and Engineering, Oxford University Press, Inc., 1998.
} 